Henri Berestycki · François Hamel · Nikolai Nadirashvili

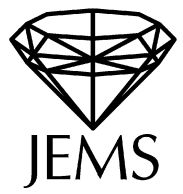

\title{
The speed of propagation for KPP type problems. I: Periodic framework
}

Received April 20, 2004

\begin{abstract}
This paper is devoted to some nonlinear propagation phenomena in periodic and more general domains, for reaction-diffusion equations with Kolmogorov-Petrovsky-Piskunov (KPP) type nonlinearities. The case of periodic domains with periodic underlying excitable media is a follow-up of the article [7]. It is proved that the minimal speed of pulsating fronts is given by a variational formula involving linear eigenvalue problems. Some consequences concerning the influence of the geometry of the domain, of the reaction, advection and diffusion coefficients are given. The last section deals with the notion of asymptotic spreading speed. The main properties of the spreading speed are given. Some of them are based on some new Liouville type results for nonlinear elliptic equations in unbounded domains.
\end{abstract}

Keywords. Reaction-diffusion equations, travelling fronts, propagation, periodic media, eigenvalue problems

\section{Contents}

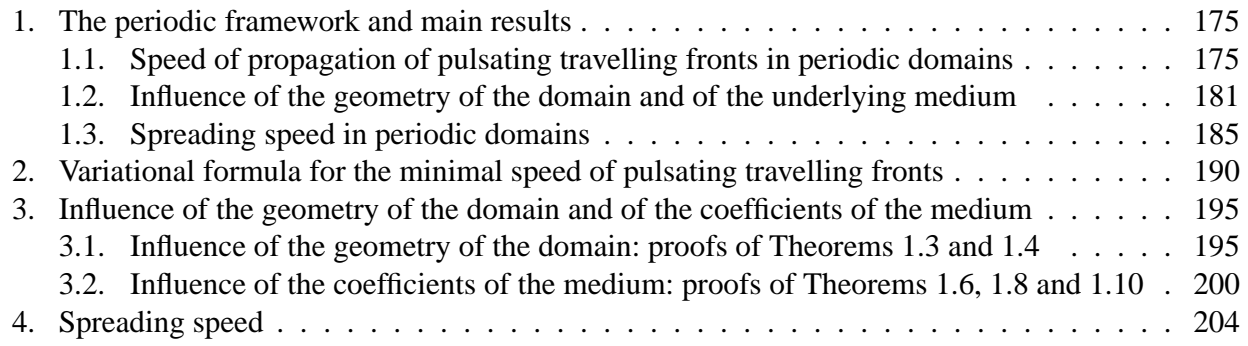

The third author was partially supported by an NSF grant.

H. Berestycki: EHESS, CAMS, 54 Boulevard Raspail, F-75006 Paris, France; e-mail: hb@ehess.fr F. Hamel: Université Aix-Marseille III, LATP, Faculté des Sciences et Techniques, Case cour A, Avenue Escadrille Normandie-Niemen, F-13397 Marseille Cedex 20, France; e-mail: francois.hamel@univ.u-3mrs.fr

Nikolai Nadirashvili: CNRS, LATP, 39 rue F. Joliot-Curie, 13453 Marseille Cedex 13, France; e-mail: nicolas@cmi.univ-mrs.fr

Mathematics Subject Classification (2000): Primary 35J60, 35K57, 35K55, 35P15; Secondary 35B10, 35B30, 35B50, 35K15 


\section{Introduction}

This paper is the first in a series of two in which we address spreading and propagation properties connected with reaction-diffusion type equations in a general framework. We consider reaction terms of the type associated with Fisher or KPP (for Kolmogorov, Petrovsky and Piskunov) equations. These properties are well understood in the homogeneous framework which we recall below. Here and in part II we consider heterogeneous problems. Part II will be devoted to propagation properties in very general domains. The present paper deals with the periodic case where both the equation and the domain have periodic structures. The precise setting and assumptions will be given shortly. But before that, let us recall some of the basic features of the homogeneous equations.

Consider the Fisher-KPP equation

$$
u_{t}-\Delta u=f(u) \quad \text { in } \mathbb{R}^{N} .
$$

It has been introduced in the celebrated papers of Fisher (1937) and KPP (1937) originally motivated by models in biology. Here the main assumption is that $f$ is say a $C^{1}$ function satisfying

$$
\begin{gathered}
f(0)=f(1)=0, \quad f^{\prime}(1)<0, \quad f^{\prime}(0)>0, \quad f>0 \text { in }(0,1), \quad f<0 \text { in }(1,+\infty), \\
f(s) \leq f^{\prime}(0) s, \quad \forall s \in[0,1] .
\end{gathered}
$$

Archetypes of such nonlinearities are $f(s)=s(1-s)$ or $f(s)=s\left(1-s^{2}\right)$.

Two fundamental features of this equation account for its success in representing propagation (or invasion) and spreading. First, this equation has a family of planar travelling fronts. These are solutions of the form

$$
u(t, x)=U(x \cdot e-c t),
$$

where $e$ is a fixed vector of unit norm which is the direction of propagation, and $c>0$ is the speed of the front. Here $U: \mathbb{R} \rightarrow \mathbb{R}$ is given by

$$
\left\{\begin{array}{l}
-U^{\prime \prime}-c U^{\prime}=f(U) \quad \text { in } \mathbb{R} \\
U(-\infty)=1, \quad U(+\infty)=0
\end{array}\right.
$$

In the original paper of Kolmogorov, Petrovsky and Piskunov, it was proved that, under the above assumptions, there is a threshold value $c^{*}=2 \sqrt{f^{\prime}(0)}>0$ for the speed $c$. Namely, no fronts exist for $c<c^{*}$, and, for each $c \geq c^{*}$, there is a unique front of the type 0.40 .5 . Uniqueness is up to shift in space or time variables.

Another fundamental property of this equation was established mathematically by Aronson and Weinberger (1978). It deals with the asymptotic speed of spreading. Namely, if $u_{0}$ is a nonnegative continuous function in $\mathbb{R}^{N}$ with compact support and $u_{0} \not \equiv 0$, then the solution $u(t, x)$ of 0.1 with initial condition $u_{0}$ at time $t=0$ spreads with speed $c^{*}$ in all directions for large times: as $t \rightarrow+\infty, \max _{|x| \leq c t}|u(t, x)-1| \rightarrow 0$ for each $c \in\left[0, c^{*}\right)$, and $\max _{|x| \geq c t} u(t, x) \rightarrow 0$ for each $c>c^{*}$. 
In this paper, we consider a general heterogeneous periodic framework extending 0.1 . The heterogeneous character arises both in the equation and in the underlying domain. The types of equations we consider here are:

$$
\left\{\begin{array}{l}
u_{t}-\nabla \cdot(A(x) \nabla u)+q(x) \cdot \nabla u=f(x, u) \quad \text { in } \Omega \\
v \cdot A \nabla u=0 \text { on } \partial \Omega
\end{array}\right.
$$

where $v$ denotes the outward unit normal on $\partial \Omega$. It will be assumed throughout this paper that the matrix $A(x)$, the vector $q(x)$ and the reaction term $f(x, u)$ as well as the geometry of $\Omega$ are periodic. Precise assumptions will be described shortly. Note that even equation 0.1 , if set in a periodic domain (e.g. the space with a periodic array of holes), acquires the features of a non-homogeneous equation. That equation will be considered in general (non-periodic) domains in Part II [10].

Here, in the periodic setting, we address three types of questions.

1) What is the speed of generalized travelling fronts in periodic structures (we recall the definition of such fronts below)? A formula which we announced in [7] is proved here.

2) Using a formula of Gärtner and Freidlin [39], we relate the asymptotic speed of spreading in a periodic domain to that of the minimal speed of propagation. In contrast to the homogeneous equation, as we will see on an example, these two speeds may not be the same.

3) We then proceed to derive several important consequences on the minimal speed of propagation and on the asymptotic spreading speed. Effects of stirring, of reaction, and of geometry will be established here rigorously. These formulas indeed allow us to prove properties of the following kind. The presence of holes or of an undulating boundary always hinders the progression or the spreading. On the contrary, any stirring by a flow always increases that speed.

In the next section we introduce the general setting with precise assumptions and we state the main results of this paper. Their proofs take up the remaining sections.

\section{The periodic framework and main results}

\subsection{Speed of propagation of pulsating travelling fronts in periodic domains}

This section deals with pulsating fronts travelling in a given unbounded periodic domain under the effects of diffusion, reaction and possibly advection by a given underlying flow. One of the most important issues in this context is the determination of the speed of propagation of fronts. A variational formula for the minimal speed of propagation is derived.

This notion of propagation of travelling fronts for the homogeneous equation $(0.1)$ can be extended to that of pulsating travelling fronts in a more general class of periodic domains and for a more general class of reaction-diffusion-advection equations in periodic excitable media.

We now describe the general periodic framework. Let $N \geq 1$ be the space dimension and let $d$ be an integer such that $1 \leq d \leq N$. Set $x=\left(x_{1}, \ldots, x_{d}\right)$ and $y=$ 
$\left(x_{d+1}, \ldots, x_{N}\right)$. Let $L_{1}, \ldots, L_{d}$ be $d$ positive numbers and let $\Omega$ be a $C^{3}$ nonempty connected open subset of $\mathbb{R}^{N}$ such that

$$
\left\{\begin{array}{l}
\exists R \geq 0, \forall(x, y) \in \Omega, \quad|y| \leq R, \\
\forall\left(k_{1}, \ldots, k_{d}\right) \in L_{1} \mathbb{Z} \times \cdots \times L_{d} \mathbb{Z}, \quad \Omega=\Omega+\sum_{i=1}^{d} k_{i} e_{i},
\end{array}\right.
$$

where $\left(e_{i}\right)_{1 \leq i \leq N}$ is the canonical basis of $\mathbb{R}^{N}$. Let $C$ be the set defined by

$$
C=\left\{(x, y) \in \Omega: x \in\left(0, L_{1}\right) \times \cdots \times\left(0, L_{d}\right)\right\} .
$$

Since $d \geq 1, \Omega$ is unbounded and $C$ is its periodicity cell. In all what follows, a field $w$ is said to be $L$-periodic with respect to $x$ in $\Omega$ if $w\left(x_{1}+k_{1}, \ldots, x_{d}+k_{d}, y\right)=$ $w\left(x_{1}, \ldots, x_{d}, y\right)$ almost everywhere in $\Omega$, for all $k=\left(k_{1}, \ldots, k_{d}\right) \in L_{1} \mathbb{Z} \times \cdots \times L_{d} \mathbb{Z}$.

Before going further on, let us point out that this framework includes several types of simpler geometrical configurations. The case of the whole space $\mathbb{R}^{N}$ corresponds to $d=N$, where $L_{1}, \ldots, L_{N}$ are any positive numbers. The case of the whole space $\mathbb{R}^{N}$ with a periodic array of holes can also be considered. The case $d=1$ corresponds to domains which have only one unbounded dimension, namely infinite cylinders which may be straight or have oscillating periodic boundaries, and which may or not have periodic holes. The case $2 \leq d \leq N-1$ corresponds to infinite slabs.

We are interested in propagation phenomena for the following reaction-diffusionadvection equation, with unknown $u$, set in the periodic domain $\Omega$ :

$$
\left\{\begin{array}{l}
u_{t}=\nabla \cdot(A(x, y) \nabla u)+q(x, y) \cdot \nabla u+f(x, y, u), \quad t \in \mathbb{R},(x, y) \in \Omega, \\
\nu A \nabla u(x, y)=0, \quad t \in \mathbb{R},(x, y) \in \partial \Omega
\end{array}\right.
$$

Such equations arise especially in simple combustion models for flame propagation [75], [90], [95], as well as in models in biology and for population dynamics [30], [69], [81]. The passive quantity $u$ typically stands for the temperature or a concentration which diffuses and is transported in a periodic excitable medium.

Let us now detail the assumptions on the coefficients of 1.2 . First, the diffusion matrix $A(x, y)=\left(A_{i j}(x, y)\right)_{1 \leq i, j \leq N}$ is a symmetric $C^{2, \delta}(\bar{\Omega})($ with $\delta>0) 1$ matrix field satisfying

$$
\left\{\begin{array}{l}
A \text { is } L \text {-periodic with respect to } x \\
\exists 0<\alpha_{1} \leq \alpha_{2}, \forall(x, y) \in \bar{\Omega}, \forall \xi \in \mathbb{R}^{N}, \quad \alpha_{1}|\xi|^{2} \leq A_{i j}(x, y) \xi_{i} \xi_{j} \leq \alpha_{2}|\xi|^{2}
\end{array}\right.
$$

\footnotetext{
1 The smoothness assumptions on $A$, as well as on $q$ and $f$ below, are made to ensure the applicability of some a priori gradient estimates for solutions of some approximating elliptic equations obtained from 1.2 (see 2.9 in Section 2). These gradient estimates are obtained for smooth $\left(C^{3}\right.$ ) solutions through a Bernstein-type method [8]. We however believe that the smoothness assumptions on $A$, as well as on $q$ and $f$, could be relaxed, by approximating $A, q$ and $f$ by smoother coefficients.
} 
(we use the usual summation convention with $1 \leq i, j \leq N$ ). The boundary term $v A \nabla u(x, y)$ stands for $v_{i}(x, y) A_{i j}(x, y) \partial_{x_{j}} u(t, x, y)$ and $v$ denotes the unit outward normal to $\Omega$. When $A$ is the identity matrix, the boundary condition reduces to the usual Neumann condition.

The underlying advection $q(x, y)=\left(q_{1}(x, y), \ldots, q_{N}(x, y)\right)$ is a $C^{1, \delta}(\bar{\Omega})$ (with $\delta>0$ ) vector field satisfying

$$
\left\{\begin{array}{l}
q \text { is } L \text {-periodic with respect to } x \\
\nabla \cdot q=0 \quad \text { in } \bar{\Omega} \\
q \cdot v=0 \quad \text { on } \partial \Omega \\
\forall 1 \leq i \leq d, \quad \int_{C} q_{i} d x d y=0
\end{array}\right.
$$

The divergence-free assumption means that the underlying flow is incompressible. The vector field $q$ is tangent to $\partial \Omega$ and its first $d$ components have been normalized. The flow $q$ may represent some turbulent fluctuations with respect to a mean field.

Lastly, let $f(x, y, u)$ be a nonnegative ${ }^{2}$ function defined in $\bar{\Omega} \times[0,1]$, such that

$$
\left\{\begin{array}{l}
f \geq 0, f \text { is } L \text {-periodic with respect to } x \text { and of class } C^{1, \delta}(\bar{\Omega} \times[0,1]), \\
\forall(x, y) \in \bar{\Omega}, \quad f(x, y, 0)=f(x, y, 1)=0, \\
\exists \rho \in(0,1), \forall(x, y) \in \bar{\Omega}, \forall 1-\rho \leq s \leq s^{\prime} \leq 1, \quad f(x, y, s) \geq f\left(x, y, s^{\prime}\right), \\
\forall s \in(0,1), \exists(x, y) \in \bar{\Omega}, \quad f(x, y, s)>0, \\
\forall(x, y) \in \bar{\Omega}, \quad f_{u}^{\prime}(x, y, 0):=\lim _{u \rightarrow 0^{+}} f(x, y, u) / u>0 .
\end{array}\right.
$$

The simplest case of such a monostable function $f(x, y, u)$ satisfying $(1.5)$ is when $f(x, y, u)=g(u)$ and the $C^{1, \delta}$ function $g$ satisfies $g(0)=g(1)=0, g>0$ on $(0,1)$, $g^{\prime}(0)>0$ and $g^{\prime}(1)<0$. Such nonlinearities arise in combustion and biological models (see Fisher [30], Kolmogorov, Petrovsky and Piskunov [57], Aronson and Weinberger [1]). Another example of such a function $f$ is $f(x, y, u)=h(x, y) \tilde{f}(u)$ where $\tilde{f}$ is as before and $h$ is $L$-periodic with respect to $x$, Lipschitz-continuous and positive in $\bar{\Omega}$.

This section is concerned with special solutions, which are called pulsating travelling fronts (or periodic travelling fronts, see [82]), and which are classical time-global solutions $u$ of $[1.2$ satisfying $0 \leq u \leq 1$ and

$$
\left\{\begin{array}{l}
\forall k \in \prod_{i=1}^{d} L_{i} \mathbb{Z}, \forall(t, x, y) \in \mathbb{R} \times \bar{\Omega}, \quad u\left(t-\frac{k \cdot e}{c}, x, y\right)=u(t, x+k, y), \\
u(t, x, y) \underset{x \cdot e \rightarrow+\infty}{\longrightarrow} 0, \quad u(t, x, y) \underset{x \cdot e \rightarrow-\infty}{\longrightarrow} 1,
\end{array}\right.
$$

\footnotetext{
2 In [7], this assumption of $f$ being nonnegative was explicit in formula (1.7) for a function $f=f(u)$ depending only on $u$. However, although this assumption was obviously also used for the general periodic nonlinearity $f(x, y, u)$ described in [7], it was not mentioned there explicitly. An extension to divergence-type equations with $f$ which may change sign is proved in [11].
} 
where the above limits hold locally in $t$ and uniformly in $y$ and in the directions of $\mathbb{R}^{d}$ which are orthogonal to $e$. Here, $e=\left(e^{1}, \ldots, e^{d}\right)$ is a given unit vector in $\mathbb{R}^{d}$. Such a solution satisfying (1.6) is then called a pulsating travelling front propagating in direction $e$. We say that $c$ is the effective unknown speed, $c \neq 0$. Let us mention here that, without the uniformity of the limits in (1.6), many other fronts may exist, whose level sets may for instance have conical shapes (see e.g. [19], [42], [43]).

Under the above assumptions, the first two authors proved in [7] that there exists $c^{*}(e)>0$ such that pulsating travelling fronts $u$ in direction $e$ with speed $c$ exist if and only if $c \geq c^{*}(e)$; furthermore, all such pulsating fronts are increasing in time $t$ (other results with more general nonlinearities $f$ were proved in [7], see below). The following theorem gives a variational characterization of this minimal speed $c^{*}(e)$ under an additional assumption on the nonlinearity $f$.

Assume that $\Omega, A$ and $q$ satisfy (1.1), (1.3) and (1.4), and that $f$ satisfies (1.5) and

$$
\forall(x, y, s) \in \bar{\Omega} \times(0,1), \quad 0<f(x, y, s) \leq f_{u}^{\prime}(x, y, 0) s .
$$

Set $\zeta(x, y):=f_{u}^{\prime}(x, y, 0)$ and $\tilde{e}=\left(e^{1}, \ldots, e^{d}, 0, \ldots, 0\right) \in \mathbb{R}^{N}$.

Theorem 1.1. Under the above assumptions, let $c^{*}(e)$ be the minimal speed of pulsating travelling fronts propagating in direction e and solving (1.2) and (1.6). Then

$$
c^{*}(e)=\min _{\lambda>0} \frac{k(\lambda)}{\lambda},
$$

where $k(\lambda)$ is the principal eigenvalue of the operator

$$
L_{\lambda} \psi:=\nabla \cdot(A \nabla \psi)-2 \lambda \tilde{e} A \nabla \psi+q \cdot \nabla \psi+\left[-\lambda \nabla \cdot(A \tilde{e})-\lambda q \cdot \tilde{e}+\lambda^{2} \tilde{e} A \tilde{e}+\zeta\right] \psi
$$

acting on the set $E=\left\{\psi \in C^{2}(\bar{\Omega}): \psi\right.$ is L-periodic with respect to $x$ and $\nu A \nabla \psi=$ $\lambda(\nu A \tilde{e}) \psi$ on $\partial \Omega\}$.

Before studying the consequences of Theorem 1.1. let us briefly explain the formula for the minimal speed $c^{*}$ and mention some earlier results about front propagation, starting from the simplest case of planar fronts in homogeneous media.

Assumption (1.7) is often called the Fisher-KPP assumption (see Fisher [32] and Kolmogorov, Petrovsky and Piskunov [57]). It is in particular satisfied for the canonical example $f(u)=u(1-u)$, or more generally when $f=f(u)$ is a $C^{2}$ concave function on $[0,1]$, positive on $(0,1)$. Thus, under the KPP assumption (1.7), the minimal speed $c^{*}(e)$ can be explicitly given in terms of $e$, the domain $\Omega$, the coefficients $q$ and $A$, and $f_{u}^{\prime}(\cdot, \cdot, 0)$. We point out that the dependence of $c^{*}(e)$ on the function $f$ is only through the $u$-derivative of $f$ at $u=0$. When $\Omega=\mathbb{R}^{N}, A=I, q=0$ and $f=f(u)$ (with $f(u) \leq f^{\prime}(0) u$ in $\left.[0,1]\right)$, formula $(1.8)$ then reduces to the well-known KPP formula $c^{*}(e)=2 \sqrt{f^{\prime}(0)}$ for the minimal speed of planar fronts for the reaction-diffusion equation $u_{t}=\Delta u+f(u)$ in $\mathbb{R}^{N}$.

A planar front is a solution of the type $\phi(x \cdot e-c t)$, where the planar profile $\phi$ solves $\phi^{\prime \prime}+c \phi^{\prime}+f(\phi)=0$ in $\mathbb{R}$ with limiting conditions $\phi(-\infty)=1$ and $\phi(+\infty)=0$. Such a solution propagates with constant speed $c$ in direction $e$ and its shape is invariant in 
the frame moving with speed $c$ in direction $e$. Many papers were devoted to such planar fronts, also for other classes of nonlinear functions $f(u)$ (see e.g. [1], [15], [30], [31], [52]). For a detailed study of planar fronts for systems of reaction-diffusion equations, we refer to the book of Volpert, Volpert and Volpert [87] and to the references therein.

Equations with periodic nonlinearities $f(x, u)$ in space dimension 1, without advection, were first considered by Shigesada, Kawasaki and Teramoto [82], and by Hudson and Zinner [50]. The notion of travelling fronts propagating with constant speed $c$ no longer holds in general and has to be replaced with the more general one of pulsating travelling fronts, as defined in (1.6) (see [82]). The profile of such a front is not invariant anymore, but, in one space dimension, the profile is periodic in time in the frame moving with speed $c$ along the direction of propagation. In [50], a formula similar to the righthand side of (1.14) in dimension 1 was given and it was proved that for any speed not smaller than the right-hand side of (1.14), pulsating travelling fronts exist. The case of a periodic nonlinearity $f(x, u)$ changing sign with respect to $x$, based on a patch invasion model in ecology, was considered in [81] and [82], and recently revisited from a rigorous mathematical and more general point of view in [11] and [12] in dimensions 1 and higher, and for more general reaction terms. Lastly, the case of periodic diffusion with bistable type nonlinearity (see $(1.11$ below) was investigated by Nakamura [71] in dimension 1 .

The case of shear flows $q=(\alpha(y), 0, \ldots, 0)$ in straight infinite cylinders $\Omega=\mathbb{R} \times \omega$ was dealt with by Berestycki, Larrouturou and Lions [13], and Berestycki and Nirenberg [17]. Under the assumption that the coefficients of equation (1.2) do not depend on the $x_{1}$ variable, the period $L_{1}$ can be an arbitrary positive number and pulsating travelling fronts reduce in this case to travelling fronts $\phi\left(x_{1}-c t, y\right)$ which move with constant instantaneous speed $c$ and keep a constant shape. Formula (1.13) was derived in this framework in [17] for the minimal speed of travelling fronts with a nonlinearity $f=f(u)$ satisfying 1.5 and (1.7). Other nonlinearities $f(u)$ were treated in [17]: for a combustion-type nonlinearity $f$ such that

$\exists \theta \in(0,1)$ (called ignition temperature),

$$
f=0 \text { on }[0, \theta] \cup\{1\}, \quad f>0 \text { on }(\theta, 1), \quad f^{\prime}(1)<0
$$

(see [52]), there exists a unique speed $c$ and a unique (up to shift in time, or equivalently in $\left.x_{1}\right)$ travelling front $\phi\left(x_{1}-c t, y\right)$; for a bistable nonlinearity $f$ such that

$$
\begin{aligned}
& \exists \theta \in(0,1), \quad f(0)=f(\theta)=f(1)=0, \\
& f<0 \text { on }(0, \theta), \quad f>0 \text { on }(\theta, 1), \quad f^{\prime}(0)<0, \quad f^{\prime}(1)<0,
\end{aligned}
$$

there still exists a unique speed $c$ and a unique (up to shift) travelling front, under the additional assumption that the section $\omega$ of the cylinder is convex. Min-max type variational formulas-involving the values of $f(u)$ for all $u \in(0,1)$ - for the unique or minimal speeds of propagation of these travelling fronts were obtained by Hamel [41] and Heinze, Papanicolaou and Stevens [49], generalizing some results for equations [40] or systems [54], [87] in dimension 1 (see also Benguria and Depassier [5] for integral formulations in dimension 1, and Coutinho and Fernandez [26], Harris, Hudson and Zinner [45] for similar problems with discrete diffusion). Several lower and upper bounds for the speeds of 
travelling fronts in infinite cylinders, as well as some asymptotics for large advection and for other regimes, were derived by Audoly, Berestycki and Pomeau [3], Berestycki [6], Constantin, Kiselev and Ryzhik [25], [56] and Heinze [48] for combustion-type and/or general positive nonlinearities $f(u)$. Rotating flows were also considered in [3] and [56], and percolating-type flows were dealt with in [56], where estimates for the more general notion of bulk burning rate (see [24]) are given. Dirichlet type boundary conditions on $\partial \Omega$, instead of Neumann conditions, were dealt with by Gardner [38] and Vega [86] in infinite cylinders. Let us also mention here that a formula similar to (1.8) for a nonlinear source term $f(u)$ of the KPP type 1.7 ) has recently been obtained by Schwetlick for a similar hyperbolic transport equation [80].

Whereas usual travelling fronts of the type $\phi\left(x_{1}-c t, y\right)$ exist in straight infinite cylinders in the case of shear flows (assuming that all coefficients in (1.2) are invariant with respect to the variable $\left.x_{1}\right)$, this is not the case anymore in infinite cylinders $\Omega=$ $\left\{\left(x_{1}, y\right): y \in \omega\left(x_{1}\right)\right\}$ with oscillating boundaries ( $\omega$ being periodic in $x_{1}$ ), even, say, for the equation $u_{t}=\Delta u+f(u)$ without advection. Such a geometrical configuration was first considered for a bistable nonlinearity $f$ by Matano [66], and the case of ondulating cylinders whose boundaries have small spatial periods with small amplitudes was recently dealt with by Lou and Matano [61].

The case of the whole space $\mathbb{R}^{N}$ with periodic diffusion and advection was first considered by Xin [91], [93] for a combustion-type nonlinearity $f$ satisfying [1.10, for which the speed of propagation of the pulsating fronts was proved to be unique in any given direction. Note that usual travelling fronts propagating with constant speed and constant shape do not exist anymore for general advection or diffusion and one has to extend these notions. The homogenization limit in $\mathbb{R}^{N}$ with coefficients having small periods was investigated by Caffarelli, Lee and Mellet [21], Freidlin [35], Heinze [46], Majda and Souganidis [63], and Xin [94]. Heinze also considered the case of the whole space with small periodic holes [47]. Freidlin [35] and Xin [94] also studied questions related to front propagation in random media.

The more general framework of periodic domains and periodic excitable media was considered by the first two authors of this paper in [7]. It was in particular proved that for a nonnegative combustion-type nonlinearity $f(x, y, u)$ satisfying the following assumptions, more general than 1.10 :

$$
\left\{\begin{array}{l}
f \text { is } L \text {-periodic with respect to } x, \\
f \text { is globally Lipschitz-continuous and is } C^{1, \delta} \text { with respect to } u \text { for some } \delta>0, \\
\exists \theta \in(0,1), \forall(x, y) \in \bar{\Omega}, \forall s \in[0, \theta] \cup\{1\}, \quad f(x, y, s)=0, \\
\exists \rho \in(0,1-\theta), \forall(x, y) \in \bar{\Omega}, \forall 1-\rho \leq s \leq s^{\prime} \leq 1, \quad f(x, y, s) \geq f\left(x, y, s^{\prime}\right), \\
\forall s \in(\theta, 1), \exists(x, y) \in \bar{\Omega}, \quad f(x, y, s)>0,
\end{array}\right.
$$

and for a given direction $e$ of $\mathbb{R}^{d}$, there exists a unique effective speed of propagation $c(e)$ and a unique (up to shift in time) pulsating travelling front $u$ satisfying (1.2) and (1.6). As already emphasized, paper [7] also gives the proof of the existence of a minimal speed $c^{*}(e)$ of propagation of pulsating fronts for a function $f$ satisfying 1.5 . Furthermore, 
under the notations of Theorem 1.1 , the inequality

$$
c^{*}(e) \geq \min _{\lambda>0} \frac{k(\lambda)}{\lambda}
$$

holds as soon as $f$ satisfies (1.5) (see Remark 1.16 in [7]). However, the question of the uniqueness, up to shift, of the fronts for any given effective speed $c \geq c^{*}(e)$ is still an open problem.

Remark 1.2 (Equivalent formulas). It can be easily checked in the general framework described above that formula $(1.8)$ can be rewritten in the following equivalent formulations:

$$
c^{*}(e)=\min \{c: \exists \lambda>0, k(\lambda)=\lambda c\}
$$

and

$$
c^{*}(e)=\min _{\lambda>0} \min _{\psi \in F} \max _{(x, y) \in \bar{\Omega}} \frac{L_{\lambda} \psi(x, y)}{\lambda \psi(x, y)},
$$

where $F=\{\psi \in E: \psi>0$ in $\bar{\Omega}\}$. Formula 1.14 is deduced from $(1.8)$ and from some characterizations of principal eigenvalues of elliptic operators ([18], [74]). We also refer to [7] for a detailed study of the above eigenvalue problems with periodic and Neumann type boundary conditions. Such operators $L_{\lambda}$ also arise in Bloch eigenvalue problems in homogenization theory (see [22], [23], [58]).

The proof of formula (1.8), which was announced in [7], is based on the methods developed in [7] and [17] (sub- and supersolutions, regularizing approximations in bounded domains). We also mention that a formula equivalent to (1.8) was recently obtained independently with different tools by Weinberger [89] for similar problems.

\subsection{Influence of the geometry of the domain and of the underlying medium}

As we have just seen, several equivalent variational formulas for the minimal speed of propagation of pulsating travelling fronts in general periodic excitable media have been given. We now analyze the influence of the geometry of the domain and of the coefficients of the medium (reaction, diffusion and advection coefficients) on the minimal speed of propagation. Since the influence of these data may be opposite, we shall investigate each of them separately.

Let us first study the influence of the geometry of the domain. Under the assumptions of the previous subsection, it easily follows from formula 1.13 that even for a homogeneous equation, due to the geometry, the minimal speed $c^{*}(e)$ depends continuously on $e$ in the unit sphere $S^{d-1}$ of $\mathbb{R}^{d}$. Note that $c^{*}(e)$ does depend on the direction $e$ in general because of the geometry of the domain and because of the spatial heterogeneity of the coefficients of equation 1.2 . This is in contrast with the homogeneous equation

$$
u_{t}=\Delta u+f(u)
$$

in the whole space $\mathbb{R}^{N}$, for which pulsating travelling fronts are actually planar travelling fronts and the minimal speed has the same value, $c^{*}(e)=2 \sqrt{f^{\prime}(0)}$ in all directions $e$ in $\mathbb{R}^{N}$. 
Let us now consider the above homogeneous equation $u_{t}=\Delta u+f(u)$, but now set in a periodic domain $\Omega \subset \mathbb{R}^{N}$ satisfying $(1.1)$. Assume that $f$ satisfies $(1.5)$ and 1.7 . If $\Omega=\mathbb{R}^{N}$, then $c^{*}(e)=2 \sqrt{f^{\prime}(0)}$ for all $e \in \mathbb{R}^{N}$ with $|e|=1$. The following statement shows that this value $2 \sqrt{f^{\prime}(0)}$ is always an upper bound whatever $\Omega$ is (provided it satisfies (1.1), and is optimal in some sense:

Theorem 1.3. Let $\Omega \subset \mathbb{R}^{N}$ satisfy $(1.1)$ and let $f=f(u)$ satisfy $(1.5)$ and $(1.7)$. Let $e=\left(e^{1}, \ldots, e^{d}\right) \in \mathbb{R}^{d}$ be such that $|e|=1$. Let $c^{*}(e)$ be the minimal speed of pulsating travelling fronts satisfying (1.15) and 1.6) together with the Neumann boundary conditions $\partial_{\nu} u=0$ on $\partial \Omega$. Then

$$
0<c^{*}(e) \leq 2 \sqrt{f^{\prime}(0)}
$$

and $c^{*}(e)=2 \sqrt{f^{\prime}(0)}$ if and only if the domain $\Omega$ is invariant in direction $\tilde{e}$, that is, $\Omega+\tau \tilde{e}=\Omega$ for all $\tau \in \mathbb{R}$, where $\tilde{e}=\left(e^{1}, \ldots, e^{d}, 0, \ldots, 0\right) \in \mathbb{R}^{N}$.

In other words, Theorem 1.3 implies that the presence of holes (perforations) in the domain always hinders the propagation, compared to the case of the whole space. Similarly, the fronts propagate strictly less rapidly in an infinite cylinder with oscillating boundaries than in a straight infinite cylinder. The homogenization limit of small holes with a combustion type nonlinearity was dealt with by Heinze [47] (see also [72] for homogenization of linear diffusion equations with small holes).

After having proved that holes make the propagation of pulsating fronts slower than in the case of the whole space $\mathbb{R}^{N}$, it is now natural to wonder whether the bigger the holes, the smaller the minimal speed $c^{*}(e)$. Actually, the answer is no in general:

Theorem 1.4. Let $N \geq 2$ and e be any unit direction in $\mathbb{R}^{N}$. Let $f=f(u)$ satisfy 1.5 , and (1.7). Then there exist some positive numbers $L_{1}, \ldots, L_{N}$, a family $\left(\Omega_{\alpha}\right)_{0 \leq \alpha<1}$ of domains satisfying (1.1) with $d=N$ and

$$
\begin{aligned}
& \Omega_{0}=\mathbb{R}^{N}, \quad \Omega_{\alpha} \supset \Omega_{\alpha^{\prime}} \quad \text { for all } 0 \leq \alpha \leq \alpha^{\prime}<1, \\
& \bigcap_{0 \leq \alpha<\alpha^{\prime}} \Omega_{\alpha}=\Omega_{\alpha^{\prime}} \quad \text { for all } 0<\alpha^{\prime}<1,
\end{aligned}
$$

such that, if $c_{\alpha}$ denotes the minimal speed $c_{\alpha}=c^{*}\left(e, \Omega_{\alpha}\right)$ of the pulsating fronts satisfying (1.15) and (1.6) in $\Omega_{\alpha}$ with Neumann boundary conditions on $\partial \Omega_{\alpha}$, then the function $\alpha \mapsto c_{\alpha}$ is continuous on $[0,1), c_{0}=2 \sqrt{f^{\prime}(0)}, c_{\alpha}<2 \sqrt{f^{\prime}(0)}$ for all $\alpha \in(0,1)$ and $c_{\alpha} \rightarrow 2 \sqrt{f^{\prime}(0)}$ as $\alpha \rightarrow 1^{-}$.

Theorem 1.4 says that the minimal speed of propagation for the homogeneous equation 1.15 may not be monotone with respect to the size of the holes. Furthermore, under the notations of Theorem 1.4, one can say that there exists at least one value of $\alpha_{0}$ in $(0,1)$ for which the minimal speed of pulsating fronts is minimal in $\Omega_{\alpha_{0}}$ among all the domains $\Omega_{\alpha}$ for $0<\alpha<1$. 
Remark 1.5. Theorem 1.3 no longer holds for equations with periodic heterogeneous coefficients even if the equation is invariant in direction $\tilde{e}$. For instance, let $\Omega^{\prime} \subset \mathbb{R}^{N-1}$ be a periodic domain satisfying $\sqrt{1.1}$ with $d \leq N-1$, and such that $\Omega^{\prime} \neq \mathbb{R}^{N-1}$. Let $\Omega=\Omega^{\prime} \times \mathbb{R}=\left\{x=\left(x^{\prime}, x_{N}\right): x^{\prime} \in \Omega^{\prime}, x_{N} \in \mathbb{R}\right\}$. Let $f(x, u)$ be a function satisfying 1.5) and (1.7), and assume that $f(x, u)$ is written as $f(x, u)=h\left(x^{\prime}\right) \tilde{f}(u)$, where $\tilde{f}$ satisfies (1.7), $0<h\left(x^{\prime}\right) \leq 1$ in $\mathbb{R}^{N-1}, h\left(x^{\prime}\right)=1$ in $\Omega^{\prime}$ and $h \neq 1$ in $\mathbb{R}^{N-1}$. Let $e=e_{N}$ be the unit vector in the $x_{N}$-direction. Then the minimal speed of propagation of pulsating fronts solving

$$
u_{t}=\Delta u+f(x, u)
$$

and 1.6 in $\Omega$, together with $\partial_{\nu} u=0$ on $\partial \Omega$, is equal to $2 \sqrt{\tilde{f}^{\prime}(0)}$. But the minimal speed for the same equation set in the whole space $\mathbb{R}^{N}$ is strictly less than $2 \sqrt{\tilde{f}^{\prime}(0)}$ (see the proof of Theorem 1.6 below for more details).

Let us now investigate the influence of the reaction coefficients on the minimal speed of propagation.

Theorem 1.6. Under the assumptions (1.1), (1.3) and (1.4), let $f=f(x, y, u)$, resp. $g=g(x, y, u)$, be a nonnegative nonlinearity satisfying (1.5) and (1.7). Let e be a unit direction in $\mathbb{R}^{d}$ and let $c^{*}(e, f)$, resp. $c^{*}(e, g)$, be the minimal speed of propagation of pulsating fronts solving (1.2) and (1.6) with nonlinearity $f$, resp. $g$.

(a) If $f_{u}^{\prime}(x, y, 0) \leq g_{u}^{\prime}(x, y, 0)$ for all $(x, y) \in \bar{\Omega}$, then $c^{*}(e, f) \leq c^{*}(e, g)$, and if moreover $f_{u}^{\prime}(x, y, 0) \not \equiv g_{u}^{\prime}(x, y, 0)$, then $c^{*}(e, f)<c^{*}(e, g)$.

(b) If $c^{*}(e, B f)$ denotes the minimal speed for the nonlinearity $B f$, with $B>0$, then $c^{*}(e, B f)$ is increasing in $B$ and

$$
\limsup _{B \rightarrow+\infty} \frac{c^{*}(e, B f)}{\sqrt{B}}<+\infty .
$$

Furthermore, if $\Omega=\mathbb{R}^{N}$ or if $\nu A \tilde{e} \equiv 0$ on $\partial \Omega$, then $\liminf _{B \rightarrow+\infty} c^{*}(e, B f) / \sqrt{B}$ $>0$.

Part (a) of Theorem 1.6 follows immediately from Theorem 1.1 (note that similar monotonicity results also hold for equations with nonlinearities changing sign, see [11], [12]). Notice that the inequality $c^{*}(e, f) \leq c^{*}(e, g)$ holds as soon as $f$ and $g$ satisfy (1.5) and $f \leq g$, even if $f$ or $g$ does not satisfy 1.7 ) (this inequality follows from the construction of the minimal speed by approximation of speeds of fronts with combustion-type nonlinearities satisfying (1.12), see [7] and Remark 1.7 below). However, the strict inequality $c^{*}(e, f)<c(e, g)$ does not hold in general if $f \leq g$ and $f \not \equiv g$, even if $f$ and $g$ satisfy (1.5) and (1.7): indeed, under these assumptions, the dependence on $f$ of the minimal speed $c^{*}(e, f)$ is only through its derivative $f_{u}^{\prime}(x, y, 0)$ at $u=0^{+}$.

The condition $v A \tilde{e} \equiv 0$ on $\partial \Omega$ holds in particular if $A \tilde{e}$ is constant and if $\Omega$ is invariant in this direction $A \tilde{e}$ (for instance, $A=I$ and $\Omega$ is a straight infinite cylinder in direction $\tilde{e}$ ). Notice that parts (a) and (b) of Theorem 1.6 obviously hold for the KPP formula $c^{*}=$ 
$2 \sqrt{B f^{\prime}(0)}$ in the case of the homogeneous equation $\sqrt{1.15}$ in $\mathbb{R}^{N}$ with nonlinearity $B f$. However, the precise asymptotic behaviour of $c^{*}(e, B f) / \sqrt{B}$ as $B \rightarrow+\infty$ is not known in general.

Lastly, part (b) also holds good if the nonlinearity $B f$ is replaced by a nonlinearity of the type $B f+f_{0}$, with given $f$ and $f_{0}$ satisfying $(1.5)$ and 1.7 .

Remark 1.7. Similar comparison properties to those in Theorem 1.6 also hold for the unique speeds $c(e, f)$ and $c(e, g)$ of the pulsating fronts solving $(1.2)$ and $(1.6)$ in the case where the nonnegative nonlinearities $f=f(x, y, u)$ and $g=g(x, y, u)$ satisfy 1.12) and are ordered. Namely, if $f \leq g$ in $\bar{\Omega} \times[0,1]$, then $c(e, f) \leq c(e, g)$. Furthermore, in this framework, one has $c(e, f)<c(e, g)$ if $f \leq g$ and $f \not \equiv g$. These facts follow easily from the proofs in [7]. However, the behaviour of $c(e, B f)$ for large $B$ is not known in this case.

The influence of advection on the speed of propagation is more difficult to analyze, because of possible interaction between the stream lines and the geometry of the domain, especially the holes. However, at least in the case where the domain is invariant in direction $\tilde{e}$, with isotropic diffusion, one can compare the speeds of propagation in direction $e$ when there is, or not, a drift term in the equation.

Theorem 1.8. Let $\Omega \subset \mathbb{R}^{N}$ be a domain satisfying 1.1 and $\Omega+\tau \tilde{e}=\Omega$ for all $\tau \in \mathbb{R}$, where $e$ is a unit vector of $\mathbb{R}^{d}$. Assume that $A=I$ and that $f=f(u)$ satisfies 1.5 , and $(1.7)$, and that $q$ satisfies 1.4 . Let $c_{q}^{*}(e)$ be the minimal speed of the pulsating fronts solving (1.2) and (1.6), with advection coefficient $q$. Then

$$
c_{q}^{*}(e) \geq c_{0}^{*}(e)=2 \sqrt{f^{\prime}(0)}
$$

and equality holds if and only if $q \cdot \tilde{e} \equiv 0$ in $\bar{\Omega}$.

Under the above assumptions, Theorem 1.8 means that the advection, or stirring, makes the propagation faster, whether the flow is a shear flow or not. Roughly speaking, the presence of turbulence in the medium increases the speed of propagation of the pulsating fronts. Furthermore, the influence of advection on the speed of propagation is minimal if and only if the advection is orthogonal to the direction of propagation.

The influence of large periodic advection, when $q$ is replaced with $B q$ with large $B$, is analyzed by the authors in [9]. The behaviour of $c_{B q}^{*}(e)$ is always at most linear in $B$ for large $B$, in a general domain $\Omega$ which satisfies (1.1) but may not be invariant in direction $\tilde{e}$. A necessary and sufficient condition for $c_{B q}^{*}(e)$ to be at least linear in $B$ is given in [9], involving the first integrals of the velocity field $q$.

Remark 1.9. It is not clear in general whether, under the assumptions of Theorem 1.8 , $c_{B q}^{*}(e)$ is nondecreasing with respect to $B>0$. However, in the case of a shear flow $q=$ $\alpha\left(x_{2}, \ldots, x_{N}\right) e_{1}$ in a straight cylinder $\Omega=\mathbb{R} \times \omega$ in direction $e_{1}$, with, say, $\omega$ bounded in $\mathbb{R}^{N-1}, \alpha \neq \equiv 0$ of class $C^{1}$ and with zero average, the first author proved in [6] that $c_{B q}^{*}\left(e_{1}\right)$ is increasing in $B>0, c_{B q}^{*}\left(e_{1}\right) / B$ is decreasing in $B>0$ and $c_{B q}^{*}\left(e_{1}\right) / B \rightarrow \rho>0$ as $B \rightarrow+\infty$. 
As far as the influence of the diffusion coefficients is concerned, one can compare the minimal speed of propagation in the case of heterogeneous diffusion with that of a homogeneous diffusion in a given direction $e$. The following theorem also gives a result on monotonicity of the speed of propagation with respect to the intensity of diffusion:

Theorem 1.10. Under the assumptions (1.1), (1.3), (1.5) and $(1.7)$, let $q=0$. Let e be a unit direction in $\mathbb{R}^{d}$.

(1) We have

$$
c^{*}(e) \leq 2 \sqrt{M_{0} M}
$$

where $M_{0}=\max _{(x, y) \in \bar{\Omega}} \zeta(x, y)$ and $M=\max _{(x, y) \in \bar{\Omega}} \tilde{e} A(x, y) \tilde{e}$. Furthermore, equality holds in 1.17 if and only if $\zeta$ and $\tilde{e} A \tilde{e}$ are constant, $\nabla \cdot(A \tilde{e}) \equiv 0$ in $\bar{\Omega}$ and $\nu A \tilde{e}=0$ on $\partial \Omega($ if $\partial \Omega \neq \emptyset)$.

(2) Assume furthermore that $f=f(u)$ depends on $u$ alone. Let $c_{\gamma}^{*}(e)$ denote the minimal speed of pulsating fronts in direction e, with diffusion matrix $\gamma$ A, where $\gamma>0$. Then $c_{\alpha}^{*}(e) \leq c_{\beta}^{*}(e)$ if $0<\alpha \leq \beta$.

As a special case of 1.17 we see that $c_{\alpha}^{*}(e) \leq C \sqrt{\alpha}$ for all $\alpha>0$, where $C$ does not depend on $\alpha>0$. Furthermore, part (2) implies that a larger diffusion speeds up the propagation.

Remark 1.11. The assumption $q=0$ was made for the sake of simplicity in the derivation of the upper bound (1.17). However, more general bounds can be obtained when $q \neq 0$. Namely, under the assumptions $(1.1), 1.3,, 1.4,, 1.5)$ and $(1.7)$, one gets as in the proof of Theorem 1.10

$$
c^{*}(e) \leq 2 \sqrt{M_{0} M}+\max _{(x, y) \in \bar{\Omega}}(-q(x, y) \cdot \tilde{e}),
$$

where $M_{0}$ and $M$ are as in Theorem 1.10 .

Lower bounds can be obtained as well, but are more restrictive. Namely, under the assumptions $1.1, \sqrt{1.3}, \sqrt{1.4}, \sqrt{1.5}$ and $\sqrt{1.7}$, assume furthermore that $\Omega=\mathbb{R}^{N}$ or $\nu A \tilde{e}=0$ on $\partial \Omega$ if $\partial \Omega \neq \emptyset$. Then

$$
c^{*}(e) \geq \min \left(m_{0} m / b,-b+2 \sqrt{m_{0} m}\right),
$$

under the convention that $m_{0} m / b=+\infty$ if $b=0$, where $m_{0}=\min _{(x, y) \in \bar{\Omega}} \zeta(x, y), m=$ $\min _{(x, y) \in \bar{\Omega}} \tilde{e} A(x, y) \tilde{e}$ and $b=\|\nabla \cdot(A \tilde{e})\|_{L^{\infty}(\Omega)}+\|q \cdot \tilde{e}\|_{L^{\infty}(\Omega)}$. The bound $[1.18)$ is proved in Section 3.2. It implies in particular that, if $q \cdot \tilde{e} \equiv 0$, then $\liminf _{\varepsilon \rightarrow 0^{+}} c_{\varepsilon}^{*}(e) / \sqrt{\varepsilon}>0$, where $c_{\varepsilon}^{*}(e)$ denotes the minimal speed of pulsating fronts in direction $e$ with diffusion matrix $\varepsilon A$.

\subsection{Spreading speed in periodic domains}

The question of stability of travelling fronts and the asymptotic convergence to travelling fronts for the solutions of Cauchy problems of the type $[1.2)$ with "front-like" initial conditions has been thoroughly studied since the pioneering paper by Kolmogorov, Petrovsky and Piskunov [57] in the one-dimensional case (see e.g. [1], [20], [28], [31], [37], [52], 
[59], [67], [78], [79], [83], [85] for other stability results in the homogeneous 1d case, [2] for the homogeneous multidimensional case, or [14], [44], [65], [76], [77] for the case of infinite cylinders with shear flows). However, few results (see [60], [70], [92]) have so far been obtained about the stability of pulsating travelling fronts in periodic media.

Another important notion is that of asymptotic speed of propagation, or spreading (see below for the precise meaning), for solutions of Cauchy problems like 1.2 with nonnegative continuous compactly supported initial condition $u_{0} \not \equiv 0$. The spreading for the homogeneous equation 1.15 in $\mathbb{R}^{N}$ was computed by Aronson and Weinberger [2]. They proved that, under the above assumptions on $u_{0}$ and if $f$ satisfies (1.5) and $\liminf _{u \rightarrow 0^{+}} f(u) / u^{1+2 / N}>03^{3}$ then

$$
\min _{|z| \leq c t} u(t, z) \rightarrow 1 \quad \text { if } 0 \leq c<c^{*} \quad \text { and } \quad \max _{|z| \leq c t} u(t, z) \rightarrow 0 \quad \text { if } c>c^{*}, \quad \text { as } t \rightarrow+\infty,
$$

where $c^{*}$ is the minimal speed of planar fronts. The speed $c^{*}$ can then also be viewed as a spreading speed (see [1], [2], [31], [51], [53], [78] for similar results with other nonlinearities $f(u)$ in dimensions 1 or higher). These spreading properties were generalized by Mallordy and Roquejoffre [65], [77] for equations with shear flows in straight infinite cylinders.

The case of a reaction-diffusion equation (1.2) without advection in the whole space $\mathbb{R}^{N}$ with periodic coefficients was considered in the important work of Gärtner and Freidlin [39] and later by Freidlin [34] in the case with advection $q$ (the proofs in [39] and [34] used probabilistic tools). Namely, under the assumptions $1.3,(1.4),(1.5) 4$ and 1.7 , if $u_{0}$ is nonnegative, continuous and compactly supported, then the solution $u(t, z)$ of 1.2 in $\mathbb{R}^{N}$ with initial condition $u_{0}$ is such that, for any unit vector $e$ in $\mathbb{R}^{N}$,

$$
\begin{aligned}
& u(t, z+c t e) \rightarrow 1 \quad \text { if } 0 \leq c<w^{*}(e), \\
& u(t, z+c t e) \rightarrow 0 \quad \text { if } c>w^{*}(e),
\end{aligned}
$$

locally in $x \in \mathbb{R}^{N}$. Furthermore, Gärtner and Freidlin derive a formula which we call the Gärtner-Freidlin formula:

$$
w^{*}(e)=\min _{\vec{\lambda} \cdot e>0} \frac{\tilde{k}(\vec{\lambda})}{\vec{\lambda} \cdot e},
$$

where $\vec{\lambda} \in \mathbb{R}^{N}$ and $\tilde{k}(\vec{\lambda})$ is the first eigenvalue of the operator

$$
L_{\vec{\lambda}}:=\nabla \cdot(A \nabla)-2 \vec{\lambda} A \nabla+q \cdot \nabla+[-\nabla \cdot(A \vec{\lambda})-q \cdot \vec{\lambda}+\vec{\lambda} \cdot A \vec{\lambda}+\zeta]
$$

with $L$-periodicity condition (as a consequence, $w^{*}( \pm 1)=c^{*}( \pm 1)$ in dimension $N=1$ ). The speed $w^{*}(e)$ can then be viewed as a ray speed in direction $e$. It follows from (1.8) that $w^{*}(e) \leq c^{*}(e)$. Notice that the latter can also be easily obtained from 1.19$)$ and the parabolic maximum principle, putting $u_{0}$ below a pulsating front moving with speed $c^{*}(e)$ in direction $e$, even if it means changing $f$ into a function $\tilde{f}$ such that $\tilde{f} \geq f$, $\tilde{f}_{u}^{\prime}(z, 0)=f_{u}^{\prime}(z, 0)$ and 1.7 holds for $\left.\tilde{f}\right)$.

\footnotetext{
3 The latter is fulfilled if $f$ satisfies $[1.7]$ as well.

4 The function $f=f(z, u)$ was actually assumed in [34] to be positive in $\mathbb{R}^{N} \times(0,1)$.
} 
Let us also mention that several works have dealt with the solutions of Cauchy problems for equations of the type (1.2), with small diffusion $\varepsilon$, together with large reaction $\varepsilon^{-1} f$, or with slowly varying flows of the type $q(\varepsilon z)$, or for equations involving more general spatio-temporal scales. Typically, the solutions of such Cauchy problems converge as $\varepsilon \rightarrow 0^{+}$to two-phase solutions of Hamilton-Jacobi type equations, separated by interfaces (see e.g. [33], [35], [36], [63], [64]). The determination of the asymptotic speed of propagation was also studied for nonlinear integral equations in dimension 1 (see [4], [27], [68], [84]), or for systems of reaction-diffusion equations in dimension 1 (see [81]).

Recently, Weinberger [89] extended the results of Gärtner and Freidlin to the general periodic framework described in [7] and here, with possible time-discrete equations. Under assumptions (1.1), (1.3), (1.4), (1.5) and (1.7), it is proved in [89] that, for any unit direction $e$ in $\mathbb{R}^{d}$, there exists $w^{*}(e)>0$ such that, if $u(t, x, y)$ solves 1.2 with a nonnegative, continuous and compactly supported initial condition $u_{0} \not \equiv 0$, then

$$
\left\{\begin{array}{ll}
u(t, x+c t e, y) \rightarrow 1 & \text { if } 0 \leq c<w^{*}(e), \\
u(t, x+c t e, y) \rightarrow 0 & \text { if } c>w^{*}(e),
\end{array} \text { as } t \rightarrow+\infty\right.
$$

locally in $(x, y)$ with respect to the points $(x, y)$ such that $(x+c t e, y) \in \bar{\Omega}$. Furthermore,

$$
\left\{\rho \xi: \xi \in S^{d-1}, 0 \leq \rho \leq w^{*}(\xi)\right\}=\left\{x \in \mathbb{R}^{d}: x \cdot \xi \leq c^{*}(\xi) \text { for all } \xi \in S^{d-1}\right\},
$$

i.e. $w^{*}(e)=\min _{\xi \in \mathbb{R}^{d}, e \cdot \xi>0} c^{*}(\xi) / e \cdot \xi$, or

$$
w^{*}(e)=\min _{\vec{\lambda} \cdot e>0} \frac{\tilde{k}(\vec{\lambda})}{\vec{\lambda} \cdot e},
$$

with $\vec{\lambda} \in \mathbb{R}^{d}$ and $\tilde{k}(\vec{\lambda})$ being the principal eigenvalue of the operator $L_{\vec{\lambda}}:=\nabla \cdot(A \nabla)-$ $2 \tilde{\lambda} A \nabla+q \cdot \nabla+[-\nabla \cdot(A \tilde{\lambda})-q \cdot \tilde{\lambda}+\tilde{\lambda} A \tilde{\lambda}+\zeta]$ acting on the set $\tilde{E}=\left\{\psi \in C^{2}(\bar{\Omega})\right.$ : $\psi$ is $L$-periodic with respect to $x$ and $\nu A \nabla \psi=(\nu A \tilde{\lambda}) \psi$ on $\partial \Omega\}$ (we set $\tilde{\lambda}=(\vec{\lambda}, 0, \ldots, 0)$ $\left.\in \mathbb{R}^{N}\right)$.

Remark 1.12. As already emphasized, it is clear from the parabolic maximum principle that $w^{*}(e) \leq c^{*}(e)$ for all $e \in S^{d-1}$. The latter could also be viewed as a consequence of $(1.8)$ and $(1.20)$ in the case of equation 1.2$)$ in $\mathbb{R}^{N}$, or from $1.22,1.23$ in the general periodic case.

The equality $w^{*}(e)=c^{*}(e)$ holds for the homogeneous isotropic equation $u_{t}=$ $\Delta u+f(u)$ in $\mathbb{R}^{N}$, for any direction $e$, but it does not hold in general. Indeed, consider the equation

$$
u_{t}=a^{2} u_{x_{1} x_{1}}+b^{2} u_{x_{2} x_{2}}+f(u) \text { in } \mathbb{R}^{2},
$$

where $a>0$ and $b>0$ are two given constants, and $f=f(u)$ satisfies 1.5 and 1.7. From the above formulas for $w^{*}(e)$ or $c^{*}(e)$, it is easy to see that, for all $\theta \in \mathbb{R}$ and $e=(\cos \theta, \sin \theta)$,

$$
w^{*}(e)=2 \sqrt{f^{\prime}(0)} \sqrt{\frac{a^{2} b^{2}}{a^{2} \sin ^{2} \theta+b^{2} \cos ^{2} \theta}}, \quad c^{*}(e)=2 \sqrt{f^{\prime}(0)} \sqrt{a^{2} \cos ^{2} \theta+b^{2} \sin ^{2} \theta}
$$


(notice that the formula for $w^{*}(e)$ could also be deduced from the case of isotropic diffusion after scaling). Hence, the equality $w^{*}(e)=c^{*}(e)$ holds here if and only if $e=( \pm 1,0)$ or $(0, \pm 1)$, or if $a=b$ (isotropic diffusion). In other words, in the case of anisotropic diffusion $(a \neq b)$, the asymptotic spreading speed is less than the minimal speed of pulsating fronts in any direction which is not an eigenvector of the diffusion matrix. Notice also that the curve $r(\theta)=w^{*}(\cos \theta, \sin \theta)$ in polar coordinates is an ellipse, while the curve $r(\theta)=c^{*}(\cos \theta, \sin \theta)$ is not an ellipse in general (but the curve $r(\theta)=\left(c^{*}(\cos \theta, \sin \theta)\right)^{-1}$ is an ellipse $)$.

Some numerical simulations with isotropic but heterogeneous diffusion have been performed in [55], confirming that the radial speed $w^{*}(e)$ may be less than the minimal speed $c^{*}(e)$ of pulsating fronts. We conjecture that, by analogy, the strict inequality $w^{*}(e)<c^{*}(e)$ may also occur in some directions $e$ in some domains with holes. However, a condition for the equality $w^{*}(e)=c^{*}(e)$ to hold or not is not known in general in the periodic setting.

In what follows, we discuss some properties of the spreading speed $w^{*}(e)$ in periodic domains. As for the minimal speed of pulsating fronts, we study the influence on the speed $w^{*}(e)$ of all the phenomena involved in problem 1.2 .

As in Theorem 1.3, let us first consider the case of the homogeneous equation 1.15 in a periodic domain $\Omega$. Since $w^{*}(e) \leq c^{*}(e)$ for any unit direction $e \in S^{d-1}$, it follows from Theorem 1.3 that $w^{*}(e) \leq 2 \sqrt{f^{\prime}(0)}$ and that, if $w^{*}(e)=2 \sqrt{f^{\prime}(0)}$, then $\Omega$ is a straight infinite cylinder in direction $\tilde{e}$. Conversely, if $\Omega$ is a straight infinite cylinder in direction $\tilde{e}$, then $c^{*}(e)=2 \sqrt{f^{\prime}(0)}$ by Theorem 1.3 . furthermore, the last equality holds for $w^{*}(e)$ as well:

Theorem 1.13. Under the assumptions (1.1) for $\Omega$ (with $d \geq 1$ ), and (1.5) and (1.7) for $f=f(u)$, let $e$ be a unit direction in $\mathbb{R}^{d}$ and $u(t, x, y)$ be the solution of 1.15 , with a given initial condition $u_{0} \neq \equiv 0$ which is nonnegative, continuous and compactly supported. Then $w^{*}(e) \leq 2 \sqrt{f^{\prime}(0)}$, and equality holds if and only if $\Omega$ is invariant in direction $\tilde{e}$.

Theorem 1.13 rests on the following Liouville type result:

Proposition 1.14. Let $\Omega$ satisfy $(1.1)$. Let $g:[0,+\infty) \rightarrow \mathbb{R}$ be a $C^{1}$ function such that $g(0)=g(1)=0, g^{\prime}(0)>0, g>0$ in $(0,1)$ and $g<0$ in $(1,+\infty)$, and let $b \in \mathbb{R}^{N}$ be such that $|b|<2 \sqrt{g^{\prime}(0)}$. Let $u$ be a classical bounded solution of

$$
\begin{cases}\Delta u+b \cdot \nabla u+g(u)=0 & \text { in } \Omega, \\ u \geq 0 & \text { in } \Omega, \\ \partial_{\nu} u=0 & \text { on } \partial \Omega .\end{cases}
$$

Then $u \equiv 0$ or $u \equiv 1$.

This result, which is of independent interest, is a Liouville type result for some solutions of semilinear elliptic equations in periodic domains. If $u$ were assumed to be $L$-periodic and not identically equal to 0 , then the conclusion $u \equiv 1$ would follow immediately from 
the strong maximum principle, since $u$ would then be bounded from below by a positive constant (see case 1 of the proof of Proposition 1.14 in Section 4). The difficultly here is that $u$ is not assumed to be $L$-periodic a priori. Let us also mention that the conclusion of Proposition 1.14 was known in the case $\Omega=\mathbb{R}^{N}$, and was proved by Aronson and Weinberger [2] by using parabolic tools (see also Remark 4.3 below). The proof of Proposition 1.14 given in Section 4 rests on some sliding arguments and on the elliptic maximum principle.

The influence of all other phenomena (reaction, diffusion and advection) is summarized in the following propositions, most of which are consequences of the results stated in Section 1.2 .

Let us start with the dependence on the reaction terms.

Proposition 1.15. Under the assumptions (1.1), (1.3), (1.4), let e be a unit direction in $\mathbb{R}^{d}$ and let $f$ and $g$ be two functions satisfying $(1.5)$ and $(1.7)$. Denote by $w^{*}(e, f)$ and $w^{*}(e, g)$ the spreading speeds in direction e for problem 1.2 with nonlinearities $f$ and $g$ respectively. If $f_{u}^{\prime}(x, y, 0) \leq g_{u}^{\prime}(x, y, 0)$ for all $(x, y) \in \bar{\Omega}$, then

$$
w^{*}(e, f) \leq w^{*}(e, g),
$$

and if moreover $f_{u}^{\prime}(x, y, 0) \not \equiv g_{u}^{\prime}(x, y, 0)$, then $w^{*}(e, f)<w^{*}(e, g)$. Hence, $w^{*}(e, B f)$ is increasing in $B>0$. Furthermore,

$$
\limsup _{B \rightarrow+\infty} \frac{w^{*}(e, B f)}{\sqrt{B}}<+\infty .
$$

Lastly, if $\Omega=\mathbb{R}^{N}$, then $\liminf _{B \rightarrow+\infty} w^{*}(e, B f) / \sqrt{B}>0$.

The next result is about the influence of stirring on propagation.

Proposition 1.16. Let $\Omega=\mathbb{R}^{N}, A=I$ and assume that $f=f(u)$ satisfies 1.5 and 1.7. For any unit vector $e$ in $\mathbb{R}^{N}$, denote by $w_{q}^{*}(e)$ the spreading speed in direction $e$, with advection term $q$ satisfying (1.4). Then

$$
w_{q}^{*}(e) \geq w_{0}^{*}(e)=2 \sqrt{f^{\prime}(0)},
$$

and the equality $w_{q}^{*}(e)=2 \sqrt{f(0)}$ holds if and only if $q \cdot e \equiv 0$ in $\mathbb{R}^{N}$.

The last proposition is concerned with the influence of the diffusion on the asymptotic spreading speed.

Proposition 1.17. Under the assumptions (1.1), (1.3), (1.5) and (1.7), let e be a unit direction in $\mathbb{R}^{d}$. Assume moreover that $q=0$. Then

$$
w^{*}(e) \leq 2 \sqrt{M_{0} M} .
$$

Furthermore, if $\Omega=\mathbb{R}^{N}$, then

$$
w^{*}(e) \geq \min \left(m_{0} \alpha_{1} / \tilde{b},-\tilde{b}+2 \sqrt{m_{0} \alpha_{1}}\right),
$$


where $\alpha_{1}$ was given in $1.3, m_{0}=\min _{x \in \mathbb{R}^{N}} f_{u}^{\prime}(x, 0)$ and

$$
\tilde{b}=\max _{x \in \mathbb{R}^{N}, \vec{\mu} \in \mathbb{R}^{N}, \vec{\mu} \neq 0}|\nabla \cdot(A(x) \vec{\mu})| /|\vec{\mu}|+\max _{x \in \mathbb{R}^{N}}|q(x)| .
$$

Lastly, if $f=f(u)$ and $w_{\gamma}^{*}(e)$ denotes the spreading speed in direction $e$, with diffusion matrix $\gamma$ A, then

$$
w_{\alpha}^{*}(e) \leq w_{\beta}^{*}(e) \quad \text { if } 0<\alpha \leq \beta .
$$

The proofs of the above propositions are sketched in Remarks 3.23 .4 in Section 3 below.

\section{Variational formula for the minimal speed of pulsating travelling fronts}

This section is devoted to the proof of formula 1.8 of Theorem 1.1 We assume all the hypotheses of Theorem 1.1 and $e$ denotes a unit vector in $\mathbb{R}^{d}$.

Let us first collect some useful properties of the first eigenvalue $k(\lambda)$ of the operator $L_{\lambda}$ given in (1.9).

Lemma 2.1. The function $\lambda \mapsto k(\lambda)$ is a convex function of $\lambda$. Furthermore, there exists a convex function $k_{0}$ such that $k_{0}(0)=k_{0}^{\prime}(0)=0$ and

$$
\forall \lambda \in \mathbb{R}, \quad 0<\min _{\bar{\Omega}} \zeta \leq \min _{\bar{\Omega}} \zeta+k_{0}(\lambda) \leq k(\lambda) \leq \max _{\bar{\Omega}} \zeta+k_{0}(\lambda)
$$

Proof. Up to a change of notations ( $q$ into $-q$, and $e$ into $-e$ ) in the equations of [7], the first eigenvalue $k(\lambda)$ of the operator $L_{\lambda}$ corresponds to the eigenvalue $-\mu_{\gamma, \zeta}(\lambda)+\lambda \gamma$ of the operator $L_{\gamma, \lambda, \zeta}+\lambda \gamma$ in Proposition 5.7 of [7]. From parts (ii) and (iii) of Proposition 5.7 of [7], it follows that

$$
\forall \lambda \in \mathbb{R}, \quad \min _{\bar{\Omega}} \zeta+k_{0}(\lambda) \leq k(\lambda) \leq \max _{\bar{\Omega}} \zeta+k_{0}(\lambda)
$$

where $k_{0}(\lambda)$ is the first eigenvalue of the operator $L_{\lambda}-\zeta$, and $k_{0}(0)=k_{0}^{\prime}(0)=0\left(k_{0}(\lambda)\right.$ corresponds to $-h(\lambda)$ in Proposition 5.7 of [7]). It follows from [7] that the function $k_{0}$ is convex. As a consequence, $k_{0}$ is nonnegative, and (2.1) follows.

Furthermore, as in [7], the first eigenvalue $k(\lambda)$ can be rewritten as

$$
k(\lambda)=\min _{\psi \in F} \max _{\bar{\Omega}} \frac{L_{\lambda} \psi}{\psi}=\min _{\tilde{\psi} \in \tilde{F}} \max _{(x, y) \in \bar{\Omega}}\left(\frac{\nabla \cdot(A \nabla \tilde{\psi})+q \cdot \nabla \tilde{\psi}}{\tilde{\psi}(x, y)}+\zeta(x, y)\right),
$$

where $F=\left\{\psi \in C^{2}(\bar{\Omega}): \psi\right.$ is $L$-periodic with respect to $x, \nu A \nabla \psi=\lambda(\nu A \tilde{e}) \psi$ on $\partial \Omega$ and $\psi>0$ in $\bar{\Omega}\}$, and

$$
\begin{aligned}
\tilde{F} & =\left\{(x, y) \mapsto \psi(x, y) e^{-\lambda x \cdot e}: \psi \in F\right\} \\
& =\left\{\tilde{\psi} \in C^{2}(\bar{\Omega}): \tilde{\psi} e^{\lambda x \cdot e} \text { is } L \text {-periodic in } x, v A \nabla \tilde{\psi}=0 \text { on } \partial \Omega \text { and } \tilde{\psi}>0 \text { in } \bar{\Omega}\right\} .
\end{aligned}
$$

It follows from the last expression for $k(\lambda)$ in $[2.2$, as in Proposition 5.7 of [7], that the function $k$ is convex with respect to $\lambda$.

The main result of this section is the following 
Proposition 2.2. If $c \in \mathbb{R}$ satisfies

$$
c>\inf \{\gamma \in \mathbb{R}: \exists \lambda>0, k(\lambda)=\lambda \gamma\},
$$

then $c>0$ and there exists a solution $u(t, x, y)$ of (1.2) and (1.6), namely $u$ is a pulsating travelling front propagating in direction e with effective speed $c$.

This proposition is proved at the end of this section. Let us now turn to the

Proof of Theorem 1.1 As already emphasized, it follows from Remark 1.16 and Section 6.4 of [7] that, for every pulsating travelling front propagating in direction $e$, with speed $c \geq c^{*}(e)$, there exists $\lambda>0$ such that $k(\lambda)=\lambda c$. Therefore,

$$
c^{*}(e) \geq \inf \{c: \exists \lambda>0, k(\lambda)=\lambda c\} .
$$

From Proposition 2.2 above, inequality 2.3 turns out to be an equality. Furthermore, the infimum is reached since for $c=c^{*}(e)$, there still exists $\lambda^{*}>0$ such that $k\left(\lambda^{*}\right)=$ $\lambda^{*} c^{*}(e)$. Finally, one concludes that

$$
\inf _{\lambda>0} \frac{k(\lambda)}{\lambda}=\inf \{c: \exists \lambda>0, k(\lambda)=\lambda c\}=c^{*}(e)=\frac{k\left(\lambda^{*}\right)}{\lambda^{*}},
$$

whence $c^{*}(e)=\min _{\lambda>0} k(\lambda) / \lambda$. That completes the proof of Theorem 1.1

Remark 2.3. Since $c^{*}(e)>0$, it follows from the above proof and Lemma 2.1 that the function $\lambda \mapsto k(\lambda) / \lambda$ is continuous on $\mathbb{R}_{+}^{*}$ and

$$
\frac{k(\lambda)}{\lambda} \rightarrow+\infty \quad \text { as } \lambda \rightarrow 0^{+}, \quad \text { and } \quad \liminf _{\lambda \rightarrow+\infty} \frac{k(\lambda)}{\lambda}>0 .
$$

Proof of Proposition 2.2. The proof follows the lines of [7] and [17], together with the additional assumption (1.7), and we just outline it.

Let $c$ be as in Proposition 2.2 and let $c^{\prime}<c$ and $\lambda^{\prime}>0$ be such that $k\left(\lambda^{\prime}\right)=\lambda^{\prime} c^{\prime}$. Let $\psi^{\prime} \in E$ be the unique (up to multiplication) positive principal eigenfunction of

$$
L_{\lambda^{\prime}} \psi^{\prime}=k\left(\lambda^{\prime}\right) \psi^{\prime} \quad \text { in } \Omega
$$

Observe that $k\left(\lambda^{\prime}\right)$ is positive from Lemma 2.1. whence $c^{\prime}$ and $c$ are positive as well.

Finding a classical $C^{2}(\mathbb{R} \times \bar{\Omega})$ solution $u(t, x, y)$ of 1.2 and $(1.6)$ is the same, up to the change of variables

$$
u(t, x, y)=\phi(x \cdot e-c t, x, y), \quad \phi(s, x, y)=u\left(\frac{x \cdot e-s}{c}, x, y\right),
$$

as proving the existence of a function $\phi \in C^{2}(\mathbb{R} \times \bar{\Omega})$ solving

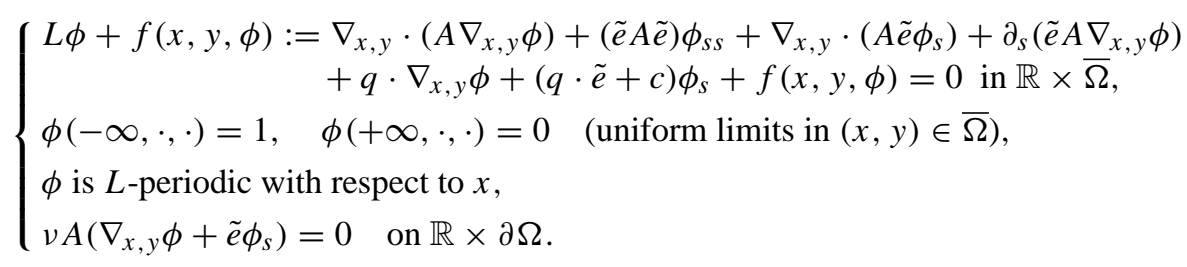


The existence of a solution $\phi$ of the above problem will be proved by solving regularized elliptic equations of the type

$$
L^{\varepsilon} \phi+f(x, y, \phi):=L \phi+\varepsilon \phi_{s s}+f(x, y, \phi)=0,
$$

where $\varepsilon>0$, in cylinders of the type $\Sigma_{a}=\{(s, x, y):-a<s<a,(x, y) \in \Omega\}$ which are bounded in the variable $s$. One then passes to the limits $a \rightarrow+\infty$ and $\varepsilon \rightarrow 0^{+}$.

To this end, first fix $a>0$. The number $\varepsilon>0$ will be chosen later. Extend $f$ by setting $f(x, y, u)=0$ for all $u \geq 1$ and $(x, y) \in \bar{\Omega}$. For $r \in \mathbb{R}$, let $v_{r}$ be the function defined by

$$
v_{r}(s, x, y)=e^{-\lambda^{\prime}(s+r)} \psi^{\prime}(x, y)
$$

for all $(s, x, y) \in \mathbb{R} \times \bar{\Omega}$. This function $v_{r}$ is a supersolution for $\varepsilon>0$ small enough and for all $r \in \mathbb{R}$, in the sense that, from 1.7 and from the definition of $\lambda^{\prime}$ and $\psi^{\prime}$,

$$
\begin{aligned}
L^{\varepsilon} v_{r}+f\left(x, y, v_{r}\right) \leq & {\left[\nabla \cdot\left(A \nabla \psi^{\prime}\right)+\left(\lambda^{\prime}\right)^{2}(\tilde{e} A \tilde{e}) \psi^{\prime}-2 \lambda^{\prime} \tilde{e} A \nabla \psi^{\prime}-\lambda^{\prime} \nabla \cdot(A \tilde{e}) \psi^{\prime}\right.} \\
& \left.+q \cdot \nabla \psi^{\prime}-\lambda^{\prime}(q \cdot \tilde{e}+c) \psi^{\prime}+\varepsilon\left(\lambda^{\prime}\right)^{2} \psi^{\prime}\right] e^{-\lambda^{\prime}(s+r)}+\zeta(x, y) v_{r} \\
\leq & {\left[k\left(\lambda^{\prime}\right)-\lambda^{\prime} c+\varepsilon\left(\lambda^{\prime}\right)^{2}\right] \psi^{\prime} e^{-\lambda^{\prime}(s+r)} } \\
\leq & \lambda^{\prime}\left(c^{\prime}-c+\varepsilon \lambda^{\prime}\right) \psi^{\prime} e^{-\lambda^{\prime}(s+r)} \leq 0
\end{aligned}
$$

as soon as $0<\varepsilon \leq\left(c-c^{\prime}\right) / \lambda^{\prime}$ (this is possible since $c^{\prime}<c$ and $\lambda^{\prime}>0$ ). Furthermore, $v_{r}$ satisfies

$$
\nu A\left(\nabla_{x, y} v_{r}+\tilde{e} \partial_{s} v_{r}\right)=\left[\nu A \nabla \psi^{\prime}-\lambda^{\prime}(\nu A \tilde{e}) \psi^{\prime}\right] e^{-\lambda^{\prime}(s+r)}=0 \quad \text { on } \mathbb{R} \times \partial \Omega
$$

because of the definition of $\psi^{\prime}$. Lastly, the function $v_{r}^{\prime}:=\min \left(v_{r}, 1\right)$ is therefore a supersolution in the above sense as well.

For all $r \in \mathbb{R}$, let $h_{r}$ be the positive constant defined by

$$
0<h_{r}:=\min _{(x, y) \in \bar{\Omega}} v_{r}^{\prime}(a, x, y) \leq 1 .
$$

The constant function $h_{r}$ clearly satisfies $L^{\varepsilon} h_{r}+f\left(x, y, h_{r}\right)=f\left(x, y, h_{r}\right) \geq 0$ in $\mathbb{R} \times \Omega$, together with $v A\left(\nabla_{x, y} h_{r}+\tilde{e} \partial_{s} h_{r}\right)=0$ on $\mathbb{R} \times \partial \Omega$. Furthermore, $h_{r} \leq v_{r}^{\prime}(s, x, y)$ for all $(s, x, y) \in \bar{\Sigma}_{a}$ since $v_{r}^{\prime}$ is nonincreasing with respect to $s$.

From the general results of Berestycki and Nirenberg [16] (see also Lemma 5.1 in [7]), there exists a solution $w_{r} \in C\left(\bar{\Sigma}_{a}\right) \cap C^{2}\left(\bar{\Sigma}_{a} \backslash\{ \pm a\} \times \partial \Omega\right)$ of

$$
\left\{\begin{array}{l}
L^{\varepsilon} w_{r}+f\left(x, y, w_{r}\right)=0 \text { in } \Sigma_{a}, \\
v A\left(\nabla_{x, y} w_{r}+\tilde{e} \partial_{s} w_{r}\right)=0 \text { on }(-a, a) \times \partial \Omega, \\
w_{r} \text { is } L \text {-periodic with respect to } x, \\
w_{r}(-a, x, y)=v_{r}^{\prime}(-a, x, y) \text { for all }(x, y) \in \bar{\Omega}, \\
w_{r}(a, \cdot, \cdot)=h_{r}, \\
0<h_{r} \leq w_{r}(s, x, y) \leq v_{r}^{\prime}(s, x, y) \text { for all }(s, x, y) \in \bar{\Sigma}_{a},
\end{array}\right.
$$

as soon as $0<\varepsilon \leq\left(c-c^{\prime}\right) / \lambda^{\prime}$. Furthermore, since $v_{r}^{\prime}$ is nonincreasing with respect to $s$ and the coefficients of $L^{\varepsilon} \cdot+f(x, y, \cdot)$ do not depend on $s$, it follows that the function $w_{r}$ 
is actually unique and nonincreasing with respect to $s$. This can be done as in Lemma 5.2 of [7], by using the same sliding method as in [16]. Lastly, the same device as in Lemma 5.3 of [7] shows that $w_{r}$ is nonincreasing with respect to $r$, and that the function $r \mapsto w_{r}$ is continuous with respect to $r$ in $C_{\text {loc }}^{2, \alpha}\left(\bar{\Sigma}_{a} \backslash\{ \pm a\} \times \partial \Omega\right.$ ) (for all $0<\alpha<1$ ) and in $C\left(\bar{\Sigma}_{a}\right)$.

Since $0 \leq h_{r} \leq w_{r} \leq v_{r}^{\prime} \leq 1$ in $\bar{\Sigma}_{a}$ and $h_{r} \rightarrow 1$ (resp. $v_{r}^{\prime} \rightarrow 0$ ) uniformly in $\bar{\Sigma}_{a}$ as $r \rightarrow-\infty$ (resp. $r \rightarrow+\infty)$, one finally concludes that, for each $\varepsilon \in\left(0,\left(c-c^{\prime}\right) / \lambda^{\prime}\right]$ and for all $a>0$, there exists a unique $r_{\varepsilon, a} \in \mathbb{R}$ such that the function $w^{\varepsilon, a}:=w_{r_{\varepsilon, a}}$ satisfies (2.4) and

$$
\max _{(x, y) \in \bar{\Omega}} w^{\varepsilon, a}(0, x, y)=1 / 2 .
$$

Let $\varepsilon \in\left(0,\left(c-c^{\prime}\right) / \lambda^{\prime}\right]$ be fixed and consider a sequence $a_{n} \rightarrow+\infty$. From the standard elliptic estimates up to the boundary, the functions $w^{\varepsilon, a_{n}}$ converge, up to taking a subsequence, in $C_{\text {loc }}^{2, \alpha}(\mathbb{R} \times \bar{\Omega})$ (for all $0<\alpha<1$ ) to a function $w^{\varepsilon}$ solving

$$
\left\{\begin{array}{l}
L^{\varepsilon} w^{\varepsilon}+f\left(x, y, w^{\varepsilon}\right)=0 \quad \text { in } \mathbb{R} \times \bar{\Omega} \\
v A\left(\nabla_{x, y} w^{\varepsilon}+\tilde{e} \partial_{s} w^{\varepsilon}\right)=0 \quad \text { on } \mathbb{R} \times \partial \Omega \\
w^{\varepsilon} \text { is } L \text {-periodic with respect to } x, \\
0 \leq w^{\varepsilon} \leq 1, \quad \max _{(x, y) \in \bar{\Omega}} w^{\varepsilon}(0, x, y)=1 / 2 .
\end{array}\right.
$$

Furthermore, $w^{\varepsilon}$ is nonincreasing with respect to $s$.

From the monotonicity of $w^{\varepsilon}$ with respect to $s$ and from the standard elliptic estimates, it follows that $w^{\varepsilon}(s, x, y) \rightarrow \phi_{ \pm}(x, y)$ in $C^{2, \alpha}(\bar{\Omega})$ as $s \rightarrow \pm \infty$, where the functions $\phi_{ \pm}$ satisfy

$$
\left\{\begin{array}{l}
\nabla \cdot\left(A \nabla \phi_{ \pm}\right)+q \cdot \nabla \phi_{ \pm}+f\left(x, y, \phi_{ \pm}\right)=0 \quad \text { in } \bar{\Omega} \\
v A \nabla \phi_{ \pm}=0 \quad \text { on } \partial \Omega \\
\phi_{ \pm} \text {is } L \text {-periodic with respect to } x \\
0 \leq \phi_{+} \leq \phi_{-} \leq 1
\end{array}\right.
$$

Integrating by parts over the cell $C$ leads to

$$
\int_{C} f\left(x, y, \phi_{ \pm}(x, y)\right) d x d y=0,
$$

whence $f\left(x, y, \phi_{ \pm}(x, y)\right) \equiv 0$ in $\bar{\Omega}$ by continuity. Now multiply the first equation of 2.6 by $\phi_{ \pm}$and integrate by parts over $C$. It follows that

$$
\int_{C} \nabla \phi_{ \pm} A \nabla \phi_{ \pm}=0
$$

and that $\phi_{ \pm}$are constants. From the monotonicity of $w^{\varepsilon}$ and the normalization of $w^{\varepsilon}$ on the section $\{0\} \times \bar{\Omega}$, together with assumption $[1.7$, , one concludes that

$$
\phi_{+}=0 \text { and } \phi_{-}=1 .
$$


Let us now come back to the variables $(t, x, y)$. For $\varepsilon \in\left(0,\left(c-c^{\prime}\right) / \lambda^{\prime}\right)$, the functions $u^{\varepsilon}$ defined by

$$
u^{\varepsilon}(t, x, y)=w^{\varepsilon}(x \cdot e-c t, x, y) \quad \text { for all }(t, x, y) \in \mathbb{R} \times \bar{\Omega}
$$

satisfy

$$
\left\{\begin{array}{l}
u_{t}^{\varepsilon}=\nabla \cdot\left(A \nabla_{x, y} u^{\varepsilon}\right)+\frac{\varepsilon}{c^{2}} u_{t t}^{\varepsilon}+q \cdot \nabla_{x, y} u^{\varepsilon}+f\left(x, y, u^{\varepsilon}\right) \quad \text { in } \mathbb{R} \times \bar{\Omega}, \\
v A \nabla_{x, y} u^{\varepsilon}=0 \quad \text { on } \mathbb{R} \times \partial \Omega, \\
\forall k \in L_{1} \mathbb{Z} \times \cdots \times L_{d} \mathbb{Z}, \forall(t, x, y) \in \mathbb{R} \times \bar{\Omega}, \quad u^{\varepsilon}\left(t-\frac{k \cdot e}{c}, x, y\right)=u^{\varepsilon}(t, x+k, y), \\
\quad \max _{x \cdot e=c t,(t, x, y) \in \mathbb{R} \times \bar{\Omega}} u^{\varepsilon}(t, x, y)=1 / 2 .
\end{array}\right.
$$

Furthermore, each $u^{\varepsilon}$ is nondecreasing in $t$ and $u^{\varepsilon}(t, x, y) \rightarrow 1$ (resp. $\rightarrow 0$ ) as $t \rightarrow+\infty$ (resp. $t \rightarrow-\infty)$ in $C_{\text {loc }}^{2}(\bar{\Omega})$.

As in Lemma 5.11 of [7], by multiplying the first equation of (2.5) by $1, w^{\varepsilon}$ and $\partial_{s} w^{\varepsilon}$ and integrating by parts over $\mathbb{R} \times C$, it follows that, for every compact set $K \subset \bar{\Omega}$, there exists a constant $C(K)$ independent of $\varepsilon$ such that

$$
\int_{\mathbb{R} \times K}\left[\left(u_{t}^{\varepsilon}\right)^{2}+\left|\nabla_{x, y} u^{\varepsilon}\right|^{2}\right] d t d x d y \leq C(K)\left(\frac{1+N\|q\|_{\infty}^{2}}{2 \alpha_{1}}+2 \max _{(x, y) \in \bar{\Omega}} F(x, y, 1)\right),
$$

where $F(x, y, t)=\int_{0}^{t} f(x, y, \tau) d \tau$ and $\alpha_{1}$ comes from 1.3 .

Let $\left(\varepsilon_{n}\right)_{n} \in\left(0,\left(c-c^{\prime}\right) / \lambda^{\prime}\right]$ be a sequence converging to $0^{+}$. There exists a function $u \in H_{\text {loc }}^{1}(\mathbb{R} \times \Omega)$ such that, up to taking a subsequence, the functions $u^{\varepsilon_{n}}$ converge, strongly in $L_{\text {loc }}^{2}(\mathbb{R} \times \Omega)$, weakly in $H_{\text {loc }}^{1}(\mathbb{R} \times \Omega)$ and almost everywhere in $\mathbb{R} \times \Omega$, to a function $u$. From parabolic regularity, the function $u$ is then a classical solution of

$$
\left\{\begin{array}{l}
u_{t}=\nabla \cdot\left(A \nabla_{x, y} u\right)+q \cdot \nabla_{x, y} u+f(x, y, u) \quad \text { in } \mathbb{R} \times \bar{\Omega}, \\
v A \nabla_{x, y} u=0 \quad \text { on } \mathbb{R} \times \partial \Omega, \\
\forall k \in L_{1} \mathbb{Z} \times \cdots \times L_{d} \mathbb{Z}, \forall(t, x, y) \in \mathbb{R} \times \bar{\Omega}, \quad u\left(t-\frac{k \cdot e}{c}, x, y\right)=u(t, x+k, y), \\
0 \leq u \leq 1 \quad \text { and } \quad u_{t} \geq 0 \quad \text { in } \mathbb{R} \times \bar{\Omega} .
\end{array}\right.
$$

Furthermore, from the normalization of $u^{\varepsilon}$ on the set $\{x \cdot e=c t\}$ and from the monotonicity of $u^{\varepsilon}$ in $t$, one has

$$
u(t, x, y) \leq 1 / 2 \text { for all }(t, x, y) \text { such that } x \cdot e \leq c t .
$$

On the other hand, (2.7) is an elliptic regularization of a parabolic equation. From Theorem A.1 of [7 5 (it is easy to check that the assumptions are satisfied, in particular the functions $u^{\varepsilon}$ are of class $C^{3}(\mathbb{R} \times \bar{\Omega})$ from the regularity assumptions and from the standard elliptic estimates), the following gradient estimates hold:

$$
\left\|\nabla_{x, y} u^{\varepsilon}\right\|_{L^{\infty}(\mathbb{R} \times \bar{\Omega})} \leq C,
$$

where $C$ is independent of $\varepsilon$.

\footnotetext{
5 We also refer to Theorem 1.6 of [8] for more general estimates for a class of elliptic regularizations of degenerate equations.
} 
Since $\max _{x \cdot e=c t} u^{\varepsilon}(t, x, y)=1 / 2$ and $u^{\varepsilon}(t-k \cdot e / c, x, y)=u^{\varepsilon}(t, x+k, y)$ in $\mathbb{R} \times \bar{\Omega}$ for all $k \in L_{1} \mathbb{Z} \times \cdots \times L_{d} \mathbb{Z}$, there exists a sequence of points $\left(t_{n}, x_{n}, y_{n}\right) \in \mathbb{R} \times \bar{C}$ such that $x_{n} \cdot e=c t_{n}$ and $u^{\varepsilon_{n}}\left(t_{n}, x_{n}, y_{n}\right)=1 / 2$. Therefore, the sequence $\left(t_{n}, x_{n}, y_{n}\right)_{n}$ is bounded and converges, up to taking a subsequence, to a point $(\bar{t}, \bar{x}, \bar{y}) \in \mathbb{R} \times \bar{C}$ such that $\bar{x} \cdot e=c \bar{t}$. Choose any $\eta>0$. From the uniform gradient estimates 2.9 , there exists $r>0$ such that $u^{\varepsilon_{n}}\left(t_{n}, x, y\right) \geq 1 / 2-\eta$ for all $n$ and for all $(x, y) \in B_{r}\left(x_{n}, y_{n}\right) \cap \bar{\Omega}$, where $B_{r}\left(x_{n}, y_{n}\right)$ denotes the euclidean closed ball in $\mathbb{R}^{N}$ of radius $r$ and centre $\left(x_{n}, y_{n}\right)$. Since each $u^{\varepsilon}$ is nondecreasing in $t$, it follows that, for $n$ large enough,

$$
u^{\varepsilon_{n}}(t, x, y) \geq 1 / 2-\eta
$$

for all $t \geq t_{n}$ and $(x, y) \in B_{r / 2}(\bar{x}, \bar{y}) \cap \bar{\Omega}$. Since $u^{\varepsilon_{n}}$ converges to the continuous function $u$ almost everywhere, one gets

$$
u(t, x, y) \geq 1 / 2-\eta
$$

for all $t \geq \bar{t}$ and for all $(x, y) \in B_{r / 2}(\bar{x}, \bar{y}) \cap \bar{\Omega}$. Since $\eta>0$ was arbitrary, it follows that $u(\bar{t}, \bar{x}, \bar{y}) \geq 1 / 2$. From 2.8 and the $(t, x)$ periodicity of $u$, one concludes that

$$
\max _{x \cdot e=c t,(t, x, y) \in \mathbb{R} \times \bar{\Omega}} u(t, x, y)=1 / 2 .
$$

Lastly, the standard parabolic estimates together with the monotonicity of $u$ with respect to $t$ imply that $u(t, x, y) \rightarrow u_{ \pm}(x, y)$ in $C_{\text {loc }}^{2}(\bar{\Omega})$ as $t \rightarrow \pm \infty$, where the functions $u_{ \pm}$satisfy

$$
\left\{\begin{array}{l}
\nabla \cdot\left(A \nabla u_{ \pm}\right)+q \cdot \nabla u_{ \pm}+f\left(x, y, u_{ \pm}\right)=0 \quad \text { in } \bar{\Omega} \\
v A \nabla u_{ \pm}=0 \quad \text { on } \partial \Omega \\
u_{ \pm} \text {are } L \text {-periodic with respect to } x
\end{array}\right.
$$

and are such that $0 \leq u_{-} \leq u_{+} \leq 1$. As explained earlier for the functions $\phi_{ \pm}$solving 2.6, one can easily prove that the functions $u_{ \pm}$are actually constant and satisfy $f\left(x, y, u_{ \pm}\right)=0$ for all $(x, y) \in \bar{\Omega}$. Furthermore, $0 \leq u_{-} \leq 1 / 2 \leq u_{+} \leq 1$ from 2.10 and $u_{t} \geq 0$. One concludes from (1.7) that $u_{-}=0$ and $u_{+}=1$.

Finally, the function $u$ is a classical solution of $(1.2)$ and (1.6). Indeed, because of the $(t, x)$ periodicity of $u$, the limits $u(t, x, y) \rightarrow 0$ (resp. $\rightarrow 1$ ) as $x \cdot e \rightarrow+\infty$ (resp. $x \cdot e \rightarrow-\infty)$ hold locally in $(t, y)$ and uniformly in the $x$ variables which are orthogonal to $e$.

That completes the proof of Proposition 2.2.

\section{Influence of the geometry of the domain and of the coefficients of the medium}

\subsection{Influence of the geometry of the domain: proofs of Theorems 1.3 and 1.4}

This subsection deals with the influence of the geometry of the domain on the speed of propagation of pulsating fronts for the homogeneous equation 1.15 in periodic domains $\Omega$. Namely, we will prove Theorems 1.3 and 1.4 
Proof of Theorem 1.3. First recall that the minimal speed $c^{*}(e)$ of the pulsating fronts solving (1.15) and (1.6) is positive (see [7]). Furthermore, from Theorem 1.1.

$$
c^{*}(e)=\min _{\lambda>0} \frac{k(\lambda)}{\lambda}
$$

where $k(\lambda)$ is the first eigenvalue of the problem

$$
\Delta \psi_{\lambda}-2 \lambda \tilde{e} \cdot \nabla \psi_{\lambda}+\left(\lambda^{2}+f^{\prime}(0)\right) \psi_{\lambda}=k(\lambda) \psi_{\lambda} \quad \text { in } \Omega
$$

and $\psi_{\lambda}$ is positive in $\bar{\Omega}, L$-periodic with respect to $x$, and satisfies $\partial_{\nu} \psi_{\lambda}=\lambda(\nu \cdot \tilde{e}) \psi_{\lambda}$ on $\partial \Omega$.

Multiply the above equation by $\psi_{\lambda}$ and integrate by parts over the cell $C$. It follows from the boundary and periodicity conditions that

$$
-\int_{C}\left|\nabla \psi_{\lambda}\right|^{2}+\left(\lambda^{2}+f^{\prime}(0)\right) \int_{C} \psi_{\lambda}^{2}=k(\lambda) \int_{C} \psi_{\lambda}^{2} .
$$

Therefore,

$$
\forall \lambda>0, \quad k(\lambda) \leq \lambda^{2}+f^{\prime}(0)
$$

and $c^{*}(e)=\min _{\lambda>0} k(\lambda) / \lambda \leq 2 \sqrt{f^{\prime}(0)}$.

Assume now that the domain $\Omega$ is invariant in direction $\tilde{e}$. Then $v \cdot \tilde{e}=0$ on $\partial \Omega$ and a (unique up to multiplication) solution $\psi_{\lambda}$ of the eigenvalue problem (3.1) is $\psi_{\lambda}=1$. Therefore, $k(\lambda)=\lambda^{2}+f^{\prime}(0)$ for all $\lambda>0$. Thus, $c^{*}(e)=2 \sqrt{f^{\prime}(0)}$.

Conversely, assume that $c^{*}(e)=2 \sqrt{f^{\prime}(0)}$. Set $\lambda^{*}=\sqrt{f^{\prime}(0)}$. We claim that $k\left(\lambda^{*}\right)=$ $\left(\lambda^{*}\right)^{2}+f^{\prime}(0)$. If not, then $k\left(\lambda^{*}\right)<\left(\lambda^{*}\right)^{2}+f^{\prime}(0)$ from 3.3 and

$$
c^{*}(e) \leq \frac{k\left(\lambda^{*}\right)}{\lambda^{*}}<\frac{\left(\lambda^{*}\right)^{2}+f^{\prime}(0)}{\lambda^{*}}=2 \sqrt{f^{\prime}(0)},
$$

which contradicts our assumption. Therefore, $k\left(\lambda^{*}\right)=\left(\lambda^{*}\right)^{2}+f^{\prime}(0)$ and it follows from (3.2) that $\psi_{\lambda^{*}}$ is constant. As a consequence, $v \cdot \tilde{e} \equiv 0$ on $\partial \Omega$. Hence, $\Omega$ is invariant in direction $\tilde{e}$.

Proof of Theorem 1.4. Up to a rotation of the frame, one can assume that $e=e_{1}=$ $(1,0, \ldots, 0)$. Furthermore, if there is a family $\left(\Omega_{\alpha}\right)_{0 \leq \alpha<1}$ of domains in $\mathbb{R}^{2}$ such that the conclusion of Theorem 1.4 holds with $N=2$ and $e=e_{1}$, then the family of domains $\left(\Omega_{\alpha}^{\prime}\right)_{0 \leq \alpha<1}=\left(\Omega_{\alpha} \times \mathbb{R}^{N-2}\right)_{0 \leq \alpha<1}$ satisfies the conclusion of Theorem 1.4 in higher dimensions $N$ with $e=e_{1}$.

Therefore, it is enough to deal with the case $N=2$ and $e=e_{1}=(1,0)$. Fix $L_{1}=$ $L_{2}=1$, and $0<\beta<1 / 2$. Let now $\left(\Omega_{\alpha}\right)_{0 \leq \alpha<1}$ be a family of smooth open connected subsets of $\mathbb{R}^{2}$ satisfying 1.1 with $L_{1}=L_{2}=1$, satisfying 1.16 and such that

$$
\forall 0 \leq \alpha \leq 1 / 3, \quad \mathbb{R}^{2} \backslash \Omega_{\alpha} \subset \mathbb{Z}^{2}+(1 / 2-\alpha, 1 / 2+\alpha) \times(1 / 2-\alpha, 1 / 2+\alpha)
$$

and

$$
\begin{aligned}
& \forall 2 / 3 \leq \alpha<1, \\
& \qquad \mathbb{Z}^{2}+(1-\alpha, \alpha) \times[\beta, 1-\beta] \subset \mathbb{R}^{2} \backslash \Omega_{\alpha} \subset \mathbb{Z}^{2}+\left(\frac{1-\alpha}{2}, \frac{1+\alpha}{2}\right) \times[\beta, 1-\beta] .
\end{aligned}
$$


One also assumes that, for each $\alpha_{0} \in(0,1)$, there exists $r>0$ such that the sets $\Omega_{\alpha}$ are $C^{3}$ uniformly with respect to $\alpha \in\left(\alpha_{0}-r, \alpha_{0}+r\right)$.

Let us prove that this family of domains satisfies the conclusion of Theorem 1.4 with $N=2$ and $e=e_{1}$.

First observe that, for each $\alpha \in(0,1)$, the domain $\Omega_{\alpha}$ is not invariant in direction $e_{1}$, whence $c_{\alpha}<2 \sqrt{f^{\prime}(0)}$ from Theorem 1.3 .

The other statements of Theorem 1.4 are proved in Steps 2, 3 and 4 below. Step 1 is concerned with the derivation of inequality 3.5 below.

Step 1. Let first $\alpha \in[0,1)$ be fixed. The minimal speed $c_{\alpha}=c^{*}\left(e_{1}, \Omega_{\alpha}\right)$ of the pulsating fronts satisfying (1.15) and (1.6) in $\Omega_{\alpha}$ with Neumann boundary conditions on $\partial \Omega_{\alpha}$ is given by the formula

$$
c_{\alpha}=\min _{\lambda>0} \frac{k_{\alpha}(\lambda)}{\lambda},
$$

where $k_{\alpha}(\lambda)$ is the first eigenvalue of the problem

$$
\Delta \psi_{\alpha, \lambda}-2 \lambda \partial_{1} \psi_{\alpha, \lambda}+\left(\lambda^{2}+f^{\prime}(0)\right) \psi_{\alpha, \lambda}=k_{\alpha}(\lambda) \psi_{\alpha, \lambda} \quad \text { in } \Omega_{\alpha}
$$

and $\psi_{\alpha, \lambda}$ is positive in $\bar{\Omega}_{\alpha},(1,1)$-periodic with respect to $\left(x_{1}, x_{2}\right)$, and satisfies $\partial_{\nu} \psi_{\alpha, \lambda}=$ $\lambda\left(v \cdot e_{1}\right) \psi_{\alpha, \lambda}$ on $\partial \Omega_{\alpha}$, where $v$ stands for the unit exterior normal to $\Omega_{\alpha}$. Observe now that, from the monotonicity of the domains $\left(\Omega_{\alpha}\right)$, one has

$$
\Omega_{\alpha} \supset \mathbb{R} \times(-\beta, \beta) .
$$

Therefore, it follows from the maximum principle (see [18] for more details) that $k_{\alpha}(\lambda) \geq$ $\kappa(\lambda)$ for all $\lambda>0$, where $\kappa(\lambda)$ (resp. $\psi_{\lambda}$ ) is the first eigenvalue (resp. eigenfunction) of

$$
\left\{\begin{array}{l}
\Delta \psi_{\lambda}-2 \lambda \partial_{1} \psi_{\lambda}+\left(\lambda^{2}+f^{\prime}(0)\right) \psi_{\lambda}=\kappa(\lambda) \psi_{\lambda} \quad \text { in } \mathbb{R} \times(-\beta, \beta), \\
\psi_{\lambda}>0 \text { in } \mathbb{R} \times(-\beta, \beta), \\
\psi_{\lambda}=0 \text { on } \mathbb{R} \times\{ \pm \beta\}, \\
\psi_{\lambda} \text { is 1-periodic with respect to } x_{1} .
\end{array}\right.
$$

By uniqueness, the function $\psi_{\lambda}$ does not depend on $x_{1}$, whence $\kappa(\lambda)=\lambda^{2}+f^{\prime}(0)-$ $(\pi /(2 \beta))^{2}$. It follows that

$$
\forall \alpha \in[0,1), \forall \lambda>0, \quad k_{\alpha}(\lambda) \geq \lambda^{2}+f^{\prime}(0)-\left(\frac{\pi}{2 \beta}\right)^{2} .
$$

Step 2. Let $\alpha$ be fixed in $(0,1)$ and let us now prove that the function $t \mapsto c_{t}$ is continuous at $\alpha$. If not, there exists $\varepsilon>0$ and a sequence $\left(\alpha_{n}\right)_{n \in \mathbb{N}} \rightarrow \alpha$ such that $\left|c_{\alpha_{n}}-c_{\alpha}\right| \geq \varepsilon$ for all $n$. Up to taking a subsequence, two cases may occur:

Case 1: $c_{\alpha_{n}} \leq c_{\alpha}-\varepsilon$ for all $n$. For each $n$, let $\lambda_{n}>0$ be such that $c_{\alpha_{n}}=k_{\alpha_{n}}\left(\lambda_{n}\right) / \lambda_{n}$ (the existence of such $\lambda_{n}$ follows from Theorem 1.1), and let $\psi_{n}=\psi_{\alpha_{n}, \lambda_{n}}$ solve (3.4) in $\Omega_{\alpha_{n}}$. The functions $\psi_{n}$ are positive in $\bar{\Omega}_{\alpha_{n}},(1,1)$-periodic in $\left(x_{1}, x_{2}\right)$ and satisfy $\partial_{\nu} \psi_{n}=$ $\lambda_{n}\left(\nu \cdot e_{1}\right) \psi_{n}$ on $\partial \Omega_{\alpha_{n}}$. Up to normalization, one can assume that $\psi_{n}(0,0)=1$. 
Since $0 \leq c_{\alpha_{n}}=k_{\alpha_{n}}\left(\lambda_{n}\right) / \lambda_{n} \leq c_{\alpha}-\varepsilon$, it follows from 3.5 that the sequence $\left(\lambda_{n}\right)$ is bounded. On the other hand, $k_{\alpha_{n}}\left(\lambda_{n}\right) \geq f^{\prime}(0)$ from Lemma 2.1. Therefore, $\left(\lambda_{n}\right)$ is bounded from below by a positive constant, so one can assume that $\lambda_{n} \rightarrow \lambda \in(0,+\infty)$ as $n \rightarrow+\infty$. On the other hand, one can also assume that $c_{\alpha_{n}} \rightarrow c \in\left[0, c_{\alpha}-\varepsilon\right]$ as $n \rightarrow+\infty$.

Furthermore, since the domains $\left(\Omega_{\alpha_{n}}\right)$ are uniformly $C^{3}$, the functions $\psi_{n}$ satisfy uniform $C^{2, \delta}$ bounds in $\bar{\Omega}_{\alpha_{n}}$ up to the boundary. Up to taking a subsequence, the functions $\psi_{n}$ converge in $C_{\text {loc }}^{2}\left(\Omega_{\alpha}\right)$ to a solution $\psi$ of

$$
\Delta \psi-2 \lambda \partial_{1} \psi+\left(\lambda^{2}+f^{\prime}(0)\right) \psi=c \lambda \psi_{\alpha, \lambda} \quad \text { in } \Omega_{\alpha},
$$

which can be extended to a $C^{2}$ function in $\bar{\Omega}_{\alpha}$ such that $\partial_{\nu} \psi=\lambda\left(v \cdot e_{1}\right) \psi$ on $\partial \Omega_{\alpha}$. Furthermore, $\psi$ is nonnegative, $(1,1)$-periodic, and satisfies $\psi(0,0)=1$. From the strong maximum principle, the function $\psi$ is positive. It is therefore the first eigenfunction of problem (3.4) with the above periodicity and boundary condition. Hence, $c \lambda=k_{\alpha}(\lambda)$. Formula (1.13) implies that $c \geq c_{\alpha}$, which contradicts the fact that $c \leq c_{\alpha}-\varepsilon$. In other words, Case 1 is ruled out.

Case 2: $c_{\alpha_{n}} \geq c_{\alpha}-\varepsilon$ for all $n$. Let now $\lambda>0$ be such that $c_{\alpha}=k_{\alpha}(\lambda) / \lambda$. From 3.3 and (1.8), one has

$$
\lambda^{2}+f^{\prime}(0) \geq k_{\alpha_{n}}(\lambda) \geq \lambda c_{\alpha_{n}} \geq \lambda\left(c_{\alpha}+\varepsilon\right) .
$$

One can assume that $k_{\alpha_{n}}(\lambda) \rightarrow k>0$ as $n \rightarrow+\infty$, and that the functions $\psi_{\alpha_{n}, \lambda}$, normalized by $\psi_{\alpha_{n}, \lambda}(0,0)=1$, converge locally in $\Omega_{\alpha}$ to a positive $(1,1)$-periodic $C^{2}\left(\bar{\Omega}_{\alpha}\right)$ solution $\psi$ of

$$
\left\{\begin{array}{l}
\Delta \psi-2 \lambda \partial_{1} \psi+\left(\lambda^{2}+f^{\prime}(0)\right) \psi=k \psi_{\alpha, \lambda} \quad \text { in } \Omega_{\alpha}, \\
\partial_{\nu} \psi=\lambda\left(\nu \cdot e_{1}\right) \psi \quad \text { on } \partial \Omega_{\alpha} .
\end{array}\right.
$$

One concludes that $k=k_{\alpha}(\lambda)$, whence $k_{\alpha}(\lambda) \geq \lambda\left(c_{\alpha}+\varepsilon\right)$ from (3.6). This contradicts the definition of $\lambda$. Therefore, Case 2 is ruled out as well.

That proves the continuity of the map $\alpha \mapsto c_{\alpha}$ in $(0,1)$.

Step 3. Let us now prove that $c_{\alpha} \rightarrow 2 \sqrt{f^{\prime}(0)}$ as $\alpha \rightarrow 0^{+}$. Assume not. Since $0 \leq c_{\alpha} \leq$ $2 \sqrt{f^{\prime}(0)}$ for all $\alpha \in[0,1)$ by Theorem 1.3 , there exists a sequence $\alpha_{n} \rightarrow 0^{+}$such that $c_{\alpha_{n}} \rightarrow c \in\left[0,2 \sqrt{f^{\prime}(0)}\right)$ as $n \rightarrow+\infty$. On the other hand, there exists a sequence $\left(\lambda_{n}\right)$ such that $c_{\alpha_{n}}=k_{\alpha_{n}}\left(\lambda_{n}\right) / \lambda_{n}$ for each $n$. As in Case 1 of Step 2 above, one can prove that the sequence $\left(\lambda_{n}\right)$ is bounded from below and above by two positive constants. From 3.3 and Lemma 2.1, it follows that $\left(k_{\alpha_{n}}\left(\lambda_{n}\right)\right)$ is itself bounded from below and above by two positive constants. Therefore, $c>0$.

For each $n$, let $u_{n}\left(t, x_{1}, x_{2}\right)$ be a pulsating travelling front solving 1.6 with speed $c_{\alpha_{n}}$ and such that

$$
\left\{\begin{array}{l}
\left(u_{n}\right)_{t}=\Delta u_{n}+f\left(u_{n}\right) \quad \text { in } \mathbb{R} \times \Omega_{\alpha_{n}} \\
\partial_{\nu} u_{n}=0 \quad \text { on } \mathbb{R} \times \partial \Omega_{\alpha_{n}} .
\end{array}\right.
$$


Furthermore, each $u_{n}$ satisfies $0 \leq u_{n} \leq 1$ and $\left(u_{n}\right)_{t} \geq 0$ in $\mathbb{R} \times \Omega_{\alpha_{n}}$. Up to normalization, one can assume that $u_{n}(0,0,0)=1 / 2$.

Owing to the construction of the domains $\Omega_{\alpha}$, and from standard parabolic estimates, the functions $u_{n}$ converge, up to taking a subsequence, to a classical solution $u=u\left(t, x_{1}, x_{2}\right)$ of

$$
u_{t}=\Delta u+f(u) \quad \text { in } \mathbb{R} \times\left(\mathbb{R}^{2} \backslash\left(\mathbb{Z}^{2}+(1 / 2,1 / 2)\right)\right)
$$

such that $0 \leq u \leq 1$. The singularities on the lines $\mathbb{R} \times\left(\mathbb{Z}^{2}+(1 / 2,1 / 2)\right)$ in $\left(t, x_{1}, x_{2}\right)$ variables are then removable and the function $u$ can be extended to a classical solution $u$ of $u_{t}=\Delta u+f(u)$ in $\mathbb{R} \times \mathbb{R}^{2}$. On the other hand, $u$ satisfies

$$
u\left(t-\frac{k_{1}}{c}, x_{1}, x_{2}\right)=u\left(t, x_{1}+k_{1}, x_{2}+k_{2}\right)
$$

for all $\left(t, x_{1}, x_{2}\right) \in \mathbb{R} \times \mathbb{R}^{2}$ and $\left(k_{1}, k_{2}\right) \in \mathbb{Z}^{2}$. Furthermore, $u_{t} \geq 0$ in $\mathbb{R} \times \mathbb{R}^{2}$ and $u(0,0,0)=1 / 2$. By passing to the limit $t \rightarrow \pm \infty$, one can prove as in $\left[7 \mid\right.$ that $u\left(t, x_{1}, x_{2}\right)$ $\rightarrow 0$ (resp. 1$)$ as $t \rightarrow-\infty$ (resp. $t \rightarrow+\infty$ ) locally in $\left(x_{1}, x_{2}\right)$.

Finally, $u$ is a pulsating travelling front, solving (1.6) with $e=e_{1}$, and (1.15) in $R \times$ $\mathbb{R}^{2}$, with speed $c$. But the minimal speed for this problem is $2 \sqrt{f^{\prime}(0)}$ (from Theorem 1.3 , the domain $\mathbb{R}^{2}$ is invariant in direction $\left.e_{1}\right)$. Therefore, $c \geq 2 \sqrt{f^{\prime}(0)}$, which contradicts our assumption.

As a consequence, the function $\alpha \mapsto c_{\alpha}$ is continuous at 0 .

Step 4. Let us now prove that $c_{\alpha} \rightarrow 2 \sqrt{f^{\prime}(0)}$ as $\alpha \rightarrow 1^{-}$. Assume not. As above, there exists a sequence $\alpha_{n} \rightarrow 1^{-}$such that $c_{\alpha_{n}} \rightarrow c \in\left(0,2 \sqrt{f^{\prime}(0)}\right)$ as $n \rightarrow+\infty$. Let $u_{n}=$ $u_{n}\left(t, x_{1}, x_{2}\right)$ be a pulsating travelling front solving (3.7) and 1.6) with speed $c_{\alpha_{n}}$. Up to normalization, one can assume that $u_{n}(0,0,0)=1 / 2$. Consider now the restrictions, still called $u_{n}$, of $u_{n}$ to $\mathbb{R} \times \mathbb{R} \times[-\beta, \beta]$. Owing to the construction of the domains $\Omega_{\alpha}$, the functions $u_{n}$ converge, up to taking a subsequence, to a classical solution $u\left(t, x_{1}, x_{2}\right)$ of

$$
\left\{\begin{array}{l}
u_{t}=\Delta u+f(u) \quad \text { in } \mathbb{R} \times(\mathbb{R} \times[-\beta, \beta] \backslash \mathbb{Z} \times\{ \pm \beta\}), \\
\partial_{\nu} u=0 \quad \text { on } \mathbb{R} \times \mathbb{R} \backslash \mathbb{Z} \times\{ \pm \beta\}
\end{array}\right.
$$

such that $0 \leq u \leq 1$. The singularities on the lines $\mathbb{R} \times \mathbb{Z} \times\{ \pm \beta\}$ in $\left(t, x_{1}, x_{2}\right)$ variables are removable and $u$ can be extended to a classical solution $u$ of $(1.15)$ in $\mathbb{R} \times \mathbb{R} \times[-\beta, \beta]$ such that $\partial_{\nu} u=0$ on $\mathbb{R} \times \mathbb{R} \times\{ \pm \beta\}$. On the other hand,

$$
u\left(t-\frac{k_{1}}{c}, x_{1}, x_{2}\right)=u\left(t, x_{1}+k_{1}, x_{2}\right)
$$

for all $\left(t, x_{1}, x_{2}\right) \in \mathbb{R} \times[-\beta, \beta]$ and $k_{1} \in \mathbb{Z}$. Furthermore, $u_{t} \geq 0$ in $\mathbb{R} \times \mathbb{R}^{2}$ and $u(0,0,0)=1 / 2$. By passing to the limit $t \rightarrow \pm \infty$, one can prove that $u\left(t, x_{1}, x_{2}\right) \rightarrow 0$ (resp. 1) as $t \rightarrow-\infty$ (resp. $t \rightarrow+\infty$ ) locally in $\left(x_{1}, x_{2}\right)$.

Finally, $u$ is a pulsating travelling front, solving $[1.15)$ in $\mathbb{R} \times \mathbb{R} \times[-\beta, \beta]$, together with Neumann boundary conditions on $\mathbb{R} \times \mathbb{R} \times\{ \pm \beta\}$. The function $u$ satisfies 1.6 
with $e=e_{1}$ and speed $c$. Since the domain $\mathbb{R} \times[-\beta, \beta]$ is invariant in direction $e_{1}$, the minimal speed of such travelling fronts is $2 \sqrt{f^{\prime}(0)}$. One then gets a contradiction with our assumption that $c<2 \sqrt{f^{\prime}(0)}$.

Therefore, $c_{\alpha} \rightarrow 2 \sqrt{f^{\prime}(0)}$ as $\alpha \rightarrow 1^{-}$and the proof of Theorem 1.4 is complete.

Remark 3.1. Let the family $\left(\Omega_{\alpha}\right)_{0 \leq \alpha<1}$ of domains in $\mathbb{R}^{2}$ be given as above. Let $e$ be a unit direction in $\mathbb{R}^{2}$ such that $e \neq \pm e_{1}$, and let $c_{\alpha}^{*}(e)$ be the minimal speed of pulsating fronts solving $\left[1.6\right.$ and 1.15 in $\Omega_{\alpha}$ with Neumann boundary conditions on $\partial \Omega_{\alpha}$. As above, the function $\alpha \mapsto c_{\alpha}^{*}(e)$ is continuous on $[0,1)$, with $c_{0}^{*}(e)=2 \sqrt{f^{\prime}(0)}$ and $c_{\alpha}^{*}(e)<2 \sqrt{f^{\prime}(0)}$ for all $\alpha \in(0,1)$. With the same arguments as above, it easily follows than $\liminf _{\alpha \rightarrow 1^{-}} c_{\alpha}^{*}(e) \geq 2 \sqrt{f^{\prime}(0)}\left|e^{1}\right|$, where $e^{1}$ is the $x_{1}$-component of the direction $e$. Nevertheless, the limit, if any, of $c_{\alpha}^{*}(e)$ as $\alpha \rightarrow 1^{-}$still remains to be determined.

\subsection{Influence of the coefficients of the medium: proofs of Theorems $1.6,1.8$ and 1.10}

This subsection is devoted to the study of the influence of the coefficients of the medium (reaction, advection and diffusion terms) on the speed of propagation of pulsating travelling fronts.

Let us first investigate the dependence on the reaction term $f$.

Proof of Theorem 1.6. (a) Let $f$ and $g$ satisfy 1.5 and 1.7 and assume that $f_{u}^{\prime}(x, y, 0)$ $\leq g_{u}^{\prime}(x, y, 0)$ for all $(x, y) \in \bar{\Omega}$. For any $\lambda>0$, let $k(\lambda, f)($ resp. $k(\lambda, g))$ be the first eigenvalue of 1.9 with $\zeta(x, y)=f_{u}^{\prime}(x, y, 0)\left(\operatorname{resp} . \zeta(x, y)=g_{u}^{\prime}(x, y, 0)\right)$. It follows from the monotonicity properties of the first eigenvalue of elliptic problems (see [18]) that $k(\lambda, f) \leq k(\lambda, g)$. Hence, Theorem 1.1 yields $c^{*}(e, f) \leq c^{*}(e, g)$.

Assume furthermore that $f_{u}^{\prime}(x, y, 0) \not \equiv g_{u}^{\prime}(x, y, 0)$. Let $\lambda_{0}>0$ be chosen so that $c^{*}(e, g)=k\left(\lambda_{0}, g\right) / \lambda_{0}$. We claim that $k\left(\lambda_{0}, f\right)<k\left(\lambda_{0}, g\right)$. If this holds, then

$$
c^{*}(e, f) \leq \frac{k\left(\lambda_{0}, f\right)}{\lambda_{0}}<\frac{k\left(\lambda_{0}, g\right)}{\lambda_{0}}=c^{*}(e, g)
$$

and we are done. Suppose then that $k\left(\lambda_{0}, f\right) \geq k\left(\lambda_{0}, g\right)$. Let $\psi_{f}$ (resp. $\psi_{g}$ ) be a positive first eigenvalue of 1.9 with $\lambda=\lambda_{0}$ and $\zeta(x, y)=f_{u}^{\prime}(x, y, 0)$ (resp. $\zeta(x, y)=$ $\left.g_{u}^{\prime}(x, y, 0)\right)$. Let $\tau>0$ be such that $\psi_{f} \leq \tau \psi_{g}$ in $\bar{\Omega}$ with equality somewhere (such a $\tau>0$ exists since both $\psi_{f}$ and $\psi_{g}$ are continuous, positive and $L$-periodic with respect to $x$ in $\bar{\Omega}$ ). The function $z:=\psi_{f}-\tau \psi_{g}$ satisfies

$$
\begin{aligned}
& \nabla \cdot(A \nabla z)-2 \lambda_{0} \tilde{e} A \nabla z+q \cdot \nabla z+\left[-\lambda_{0} \nabla \cdot(A \tilde{e})-\lambda_{0} q \cdot \tilde{e}+\lambda_{0}^{2} \tilde{e} A \tilde{e}+f_{u}^{\prime}(x, y, 0)-k\left(\lambda_{0}, f\right)\right] z \\
& \quad=\left(k\left(\lambda_{0}, f\right)-k\left(\lambda_{0}, g\right)\right) \tau \psi_{g}+\left(g_{u}^{\prime}(x, y, 0)-f_{u}^{\prime}(x, y, 0)\right) \tau \psi_{g} \geq, \not \equiv 0 \text { in } \Omega
\end{aligned}
$$

from our assumptions. On the other hand, $z \leq 0$ in $\bar{\Omega}$ with equality somewhere, and $v A \nabla z=\lambda_{0}(\nu A \tilde{e}) z$ on $\partial \Omega$. The strong maximum principle and Hopf lemma imply that $z \equiv 0$ in $\bar{\Omega}$. But the left-hand side of 3.8 is not identically zero. This contradiction yields $k\left(\lambda_{0}, f\right)<k\left(\lambda_{0}, g\right)$. 
(b) Let $f$ satisfy (1.5) and 1.7). First, it follows from part (a) that the function $B \mapsto c^{*}(e, B f)$ is increasing with respect to $B>0$. For any $\lambda>0$ and $B>0$, let $k(\lambda, B)$ and $\psi_{\lambda, B}$ be the first eigenvalue and eigenfunction of operator (1.9) with $\zeta(x, y)=B f_{u}^{\prime}(x, y, 0)$. Multiply the equation $L_{\lambda} \psi_{\lambda, B}=k(\lambda, B) \psi_{\lambda, B}$ by $\psi_{\lambda, B}$ and integrate by parts over $C$ to get

$$
\begin{aligned}
k(\lambda, B) \int_{C} \psi_{\lambda, B}^{2}= & -\int_{C} \nabla \psi_{\lambda, B} A \nabla \psi_{\lambda, B}-\lambda \int_{C}(q \cdot \tilde{e}) \psi_{\lambda, B}^{2}+\lambda^{2} \int_{C}(\tilde{e} A \tilde{e}) \psi_{\lambda, B}^{2} \\
& +\int_{C} B f_{u}^{\prime}(x, y, 0) \psi_{\lambda, B}^{2} .
\end{aligned}
$$

It follows that

$$
k(\lambda, B) \leq \lambda\|q \cdot \tilde{e}\|_{\infty}+\lambda^{2}\|\tilde{e} A \tilde{e}\|_{\infty}+B\left\|f_{u}^{\prime}(\cdot, \cdot, 0)\right\|_{\infty} .
$$

Hence,

$$
c^{*}(e, B f)=\min _{\lambda>0} \frac{k(\lambda, B)}{\lambda}=O(\sqrt{B}) \quad \text { as } B \rightarrow+\infty .
$$

Assume now that $\Omega=\mathbb{R}^{N}$ or $\nu A \cdot \tilde{e} \equiv 0$ on $\partial \Omega$. In both cases, integrating over $C$ the equation $L_{\lambda} \psi_{\lambda, B}=k(\lambda, B) \psi_{\lambda, B}$ with $\zeta(x, y)=B f_{u}^{\prime}(x, y, 0)$ leads to

$$
\begin{aligned}
k(\lambda, B) \int_{C} \psi_{\lambda, B}= & \lambda \int_{C} \nabla \cdot(A \tilde{e}) \psi_{\lambda, B}-\lambda \int_{C}(q \cdot \tilde{e}) \psi_{\lambda, B}+\lambda^{2} \int_{C}(\tilde{e} A \tilde{e}) \psi_{\lambda, B} \\
& +\int_{C} B f_{u}^{\prime}(x, y, 0) \psi_{\lambda, B} .
\end{aligned}
$$

Hence,

$$
k(\lambda, B) \geq-\lambda\|\nabla \cdot(A \tilde{e})\|_{\infty}-\lambda\|q \cdot \tilde{e}\|_{\infty}+\lambda^{2} \alpha_{1}+B \min _{(x, y) \in \bar{\Omega}} f_{u}^{\prime}(x, y, 0),
$$

where $\alpha_{1}>0$ is given in 1.3 . Since $\min _{(x, y) \in \bar{\Omega}} f_{u}^{\prime}(x, y, 0)>0$, it follows that there exists $\gamma>0$ such that

$$
c^{*}(e, B f)=\min _{\lambda>0} \frac{k(\lambda, B)}{\lambda} \geq \gamma \sqrt{B}
$$

for $B$ large enough. That completes the proof of Theorem 1.6

Remark 3.2. With the same tools as above, it can easily be seen that part (a) of Theorem 1.6 extends to the ray speed $w^{*}(e)$, as defined in Section 1.3 and in $(1.23)$. Similarly, with obvious notations, $w^{*}(e, B f) \leq c^{*}(e, B f)=O(\sqrt{B})$ as $B \rightarrow+\infty$, and $\liminf _{B \rightarrow+\infty} w^{*}(e, B f) / \sqrt{B}>0$ if $\Omega=\overline{\mathbb{R}}^{N}$.

That corresponds to Proposition 1.15 .

Proof of Theorem 1.8. Under the assumptions of Theorem 1.8, one has $v \cdot \tilde{e}=0$ on $\partial \Omega$, and $c_{q}^{*}(e)$ is given by the formula

$$
c_{q}^{*}(e)=\min _{\lambda>0} \frac{k_{q}(\lambda)}{\lambda},
$$


where $k_{q}(\lambda)$ and $\psi_{\lambda, q}$ denote the unique eigenvalue and positive $L$-periodic eigenfunction of

$\Delta \psi_{\lambda, q}-2 \lambda \tilde{e} \cdot \nabla \psi_{\lambda, q}+q \cdot \nabla \psi_{\lambda, q}+\left[-\lambda q \cdot \tilde{e}+\lambda^{2}+f^{\prime}(0)\right] \psi_{\lambda, q}=k_{q}(\lambda) \psi_{\lambda, q} \quad$ in $\Omega$

with $v \cdot \nabla \psi_{\lambda, q}=0$ on $\partial \Omega$. Divide the following formula by $\psi_{\lambda, q}$ and integrate by parts over $C$. It follows from 1.4 and the $L$-periodicity of $q$ and $\psi_{\lambda, q}$ that

$$
\int_{C} \frac{\left|\nabla \psi_{\lambda, q}\right|^{2}}{\psi_{\lambda, q}^{2}}+\left(\lambda^{2}+f^{\prime}(0)\right)|C|=k_{q}(\lambda)|C|
$$

whence

$$
k_{q}(\lambda) \geq \lambda^{2}+f^{\prime}(0)=k_{0}(\lambda) .
$$

Therefore, $c_{q}^{*}(e) \geq 2 \sqrt{f^{\prime}(0)}=c_{0}^{*}(e)$.

If $q \cdot \tilde{e} \equiv 0$, then $\psi_{\lambda, q}$ is constant for each $\lambda>0$, whence $k_{q}(\lambda)=\lambda^{2}+f^{\prime}(0)$ and $c_{q}^{*}(e)=2 \sqrt{f^{\prime}(0)}=c_{0}^{*}(e)$.

Assume now that $c_{q}^{*}(e)=c_{0}^{*}(e)=2 \sqrt{f^{\prime}(0)}$. Let $\lambda^{*}>0$ be such that

$$
c_{q}^{*}(e)=\frac{k_{q}\left(\lambda^{*}\right)}{\lambda^{*}} .
$$

Then $k_{q}\left(\lambda^{*}\right)=2 \lambda^{*} \sqrt{f^{\prime}(0)}$, whereas 3.11 yields $k_{q}\left(\lambda^{*}\right) \geq\left(\lambda^{*}\right)^{2}+f^{\prime}(0)$. Therefore, $\lambda^{*}=\sqrt{f^{\prime}(0)}$ and $k_{q}\left(\lambda^{*}\right)=\left(\lambda^{*}\right)^{2}+f^{\prime}(0)$. From 3.10 , one finds that $\psi_{\lambda^{*}, q}$ is constant, and from 3.9 one concludes that $q \cdot \tilde{e} \equiv 0$ in $\bar{\Omega}$.

Remark 3.3. With the same arguments as above and with obvious notations, one can deduce from 1.20 that, if $\Omega=\mathbb{R}^{N}, A=I$ and $f=f(u)$ satisfies 1.5 , and 1.7 , then $w_{q}^{*}(e) \geq w_{0}^{*}(e)=2 \sqrt{f^{\prime}(0)}$ for every unit vector $e$ of $\mathbb{R}^{N}$. Furthermore, the equality $w_{q}^{*}(e)=2 \sqrt{f^{\prime}(0)}$ holds if and only if $q \cdot e \equiv 0$. That corresponds to Proposition 1.16 .

Proof of Theorem 1.10. Under the assumptions of Theorem 1.10, let $k(\lambda)$ and $\psi_{\lambda}$ be the first eigenvalue and eigenfunction of the operator $L_{\lambda}$ defined in (1.9). Multiply the equation $L_{\lambda} \psi_{\lambda}=k(\lambda) \psi_{\lambda}$ by $\psi_{\lambda}$ and integrate over $C$. One gets

$$
k(\lambda) \int_{C} \psi_{\lambda}^{2}=-\int_{C} \nabla \psi_{\lambda} A \nabla \psi_{\lambda}+\lambda^{2} \int_{C} \tilde{e} A \tilde{e} \psi_{\lambda}^{2}+\int_{C} \zeta(x, y) \psi_{\lambda}^{2} .
$$

Hence,

$$
k(\lambda) \leq \max _{(x, y) \in \bar{\Omega}} \zeta(x, y)+\lambda^{2} \max _{(x, y) \in \bar{\Omega}} \tilde{e} A(x, y) \tilde{e}
$$

and (1.17) follows from (1.8).

Assume now that $\tilde{e} A(x, y) \tilde{e}=M$ and $\zeta(x, y)=M_{0}$ are constant in $\bar{\Omega}$, and that $\nabla \cdot(A \tilde{e}) \equiv 0$ in $\bar{\Omega}$ and $\nu A \tilde{e}=0$ on $\partial \Omega$ (if $\partial \Omega \neq \emptyset$ ). Then $\psi_{\lambda}$ is constant for each $\lambda>0$, whence $k(\lambda)=M_{0}+\lambda^{2} M$ and $c^{*}(e)=2 \sqrt{M_{0} M}$.

Assume now that $c^{*}(e)=2 \sqrt{M_{0} M}$, where $M_{0}=\max _{(x, y) \in \bar{\Omega}} \zeta(x, y)$ and $M=$ $\max _{(x, y) \in \bar{\Omega}} \tilde{e} A(x, y) \tilde{e}$. Let $\lambda^{*}=\sqrt{M_{0} / M}>0$. It follows from 1.8 that $k\left(\lambda^{*}\right) \geq$ 
$c^{*}(e) \lambda^{*}=2 M_{0}$. On the other hand, $k\left(\lambda^{*}\right) \leq M_{0}+\left(\lambda^{*}\right)^{2} M=2 M_{0}$ from 3.13). Therefore, $k\left(\lambda^{*}\right)=2 M_{0}=M_{0}+\left(\lambda^{*}\right)^{2} M$. One deduces from 3.12 and the equation $L_{\lambda^{*}} \psi_{\lambda^{*}}=k\left(\lambda^{*}\right) \psi_{\lambda^{*}}$ that $\psi_{\lambda^{*}}$ is constant, $\zeta(x, y) \equiv M_{0}, \tilde{e} A(x, y) \tilde{e} \equiv M, \nabla \cdot(A \tilde{e}) \equiv 0$, and $\nu A \tilde{e}=0$ on $\partial \Omega$.

Assume now that $0<\alpha \leq \beta$, and let $c_{\alpha}^{*}(e)\left(\right.$ resp. $\left.c_{\beta}^{*}(e)\right)$ denote the minimal speed of pulsating fronts in direction $e$ with diffusion $\alpha A$ (resp. $\beta A$ ). By $(1.8)$, one has

$$
c_{\alpha}^{*}(e)=\min _{\lambda>0} \frac{k^{\alpha}(\lambda)}{\lambda} \quad \text { and } \quad c_{\beta}^{*}(e)=\min _{\lambda>0} \frac{k^{\beta}(\lambda)}{\lambda},
$$

where $k^{\alpha}(\lambda)\left(\right.$ resp. $\left.k^{\beta}(\lambda)\right)$ is the first eigenvalue of the operator $\alpha \tilde{L}_{\lambda}+f^{\prime}(0)$ (resp. $\beta \tilde{L}_{\lambda}+$ $\left.f^{\prime}(0)\right)$ and $\tilde{L}_{\lambda}$ is the operator $\tilde{L}_{\lambda}=\nabla \cdot(A \nabla)-2 \lambda \tilde{e} A \nabla-\lambda \nabla \cdot(A \tilde{e})+\lambda^{2} \tilde{e} A \tilde{e}$ acting on the set $E$ ( $E$ has been defined in Theorem 1.1). Under the notations of Lemma 2.1, $k_{0}(\lambda)$ is the first eigenvalue of $\tilde{L}_{\lambda}$, whence

$$
k^{\alpha}(\lambda)=\alpha k_{0}(\lambda)+f^{\prime}(0) \quad \text { and } \quad k^{\beta}(\lambda)=\beta k_{0}(\lambda)+f^{\prime}(0)
$$

for all $\lambda>0$. On the other hand, it follows from Lemma 2.1 that the function $k_{0}$ is nonnegative. Therefore, $k^{\alpha}(\lambda) \leq k^{\beta}(\lambda)$ and 3.14 yields $c_{\alpha}^{*}(e) \leq c_{\beta}^{*}(e)$. That completes the proof of Theorem 1.10

Proof of the lower bound (1.18). Under the notations in Remark 1.11 integrate the equation $L_{\lambda} \psi_{\lambda}=k(\lambda) \psi_{\lambda}$ over $C$. It follows that

$$
k(\lambda) \int_{C} \psi_{\lambda}=\lambda \int_{C} \nabla \cdot(A \tilde{e}) \psi_{\lambda}-\lambda \int_{C} q \cdot \tilde{e} \psi_{\lambda}+\lambda^{2} \int_{C} \tilde{e} A \tilde{e} \psi_{\lambda}+\int_{C} \zeta(x, y) \psi_{\lambda} .
$$

Therefore, $k(\lambda) \geq-\lambda b+\lambda^{2} m+m_{0}$. On the other hand, Lemma 2.1 yields $k(\lambda) \geq m_{0}$. Now (1.18) easily follows from (1.8).

Remark 3.4. Under the assumptions and notations of Theorem $1.10, w^{*}(e) \leq c^{*}(e) \leq$ $2 \sqrt{M_{0} M}$ for all unit vectors $e$ in $\mathbb{R}^{d}$. Furthermore, under the additional assumption that $f=f(u)$, the ray speed $w_{\gamma}^{*}(e)$ in direction $e$ for problem 1.2 with diffusion matrix $\gamma A$ $(\gamma>0)$ is given by

$$
w_{\gamma}^{*}(e)=\min _{\vec{\lambda} \in \mathbb{R}^{d}, \vec{\lambda} \cdot e>0} \frac{\tilde{k}^{\gamma}(\vec{\lambda})}{\vec{\lambda} \cdot e},
$$

where $\tilde{k}^{\gamma}(\vec{\lambda})=\gamma k_{0, \vec{\lambda} /|\vec{\lambda}|}(|\vec{\lambda}|)+f^{\prime}(0)$ and, for all unit vectors $e^{\prime}$ in $\mathbb{R}^{d}$ and all $\mu>0$, $k_{0, e^{\prime}}(\mu)$ denotes the first eigenvalue of the operator $\nabla \cdot(A \nabla \psi)-2 \mu e^{\prime} A \nabla \psi-$ $\mu \nabla \cdot\left(A e^{\prime}\right) \psi+\mu^{2} e^{\prime} A e^{\prime} \psi$ acting on the space of $L$-periodic functions $\psi$ such that $\nu A \nabla \psi=$ $\mu\left(\nu A e^{\prime}\right) \psi$ on $\partial \Omega$. Lemma 2.1 yields $k_{0, e^{\prime}}(\mu) \geq 0$. Therefore, $w_{\alpha}^{*}(e) \leq w_{\beta}^{*}(e)$ if $0<\alpha$ $\leq \beta$.

Lastly, if $\Omega=\mathbb{R}^{N}$, then with the same arguments as above, it easily follows from 1.20 that $w^{*}(e) \geq \min \left(m_{0} \alpha_{1} / \tilde{b},-\tilde{b}+2 \sqrt{m_{0} \alpha_{1}}\right)$, where $\alpha_{1}$ was given in $1.3, m_{0}=$ $\min _{x \in \mathbb{R}^{N}} f_{u}^{\prime}(x, 0)$ and $\tilde{b}=\max _{x \in \mathbb{R}^{N}, \vec{\mu} \in \mathbb{R}^{N}, \vec{\mu} \neq 0}|\nabla \cdot(A(x) \vec{\mu})| /|\vec{\mu}|+\max _{x \in \mathbb{R}^{N}}|q(x)|$. That corresponds to Proposition 1.17 . 


\section{Spreading speed}

This section is devoted to the proof of Theorem 1.13 . It is based on the following auxiliary Lemmas 4.1 and 4.2, and on Proposition 1.14

Lemma 4.1. Let $\Omega$ satisfy 1.1 with $d \geq 1$. Let $\lambda_{R}$ be the first eigenvalue, and $\psi_{R}$ be the first eigenfunction of

$$
\begin{cases}-\Delta \psi_{R}=\lambda_{R} \psi_{R} & \text { in } \Omega \cap B_{R} \\ \psi_{R}>0 & \text { in } \bar{\Omega} \cap B_{R} \\ \psi_{R}=0 & \text { on } \bar{\Omega} \cap \partial B_{R} \\ \partial_{\nu} \psi_{R}=0 & \text { on } \partial \Omega \cap B_{R} \\ \left\|\psi_{R}\right\|_{L^{\infty}\left(\Omega \cap B_{R}\right)}=1, & \end{cases}
$$

where $B_{R}$ is the open euclidean ball of radius $R>0$ and centre 0 . Then $\lambda_{R} \rightarrow 0$ as $R \rightarrow+\infty$.

Proof. It follows from the maximum principle that $\lambda_{R}$ is decreasing with respect to $R$. Furthermore, $\lambda_{R}$ has the following variational representation:

$$
\lambda_{R}=\min _{\psi \in H^{1}\left(\Omega \cap B_{R}\right) \backslash\{0\}, \psi_{\mid \bar{\Omega} \cap \partial B_{R}}=0} \frac{\int_{\Omega \cap B_{R}}|\nabla \psi|^{2}}{\int_{\Omega \cap B_{R}} \psi^{2}} \geq 0 .
$$

Let $\xi$ be a given $C^{\infty}\left(\mathbb{R}^{N}\right)$ function such that $\xi=1$ in $B_{1 / 2}$ and $\xi=0$ in $\mathbb{R}^{N} \backslash B_{1}$. Taking the function $\psi(z)=\xi(z / R)$ as a test function leads to

$$
\lambda_{R} \leq \frac{R^{-2}\|\nabla \xi\|_{\infty}^{2}\left|\Omega \cap B_{R}\right|}{\left|\Omega \cap B_{R / 2}\right|} .
$$

But $\left|\Omega \cap B_{R}\right| /\left|\Omega \cap B_{R / 2}\right|$ is bounded as $R \rightarrow+\infty$ because of $(1.1)$, whence $\lambda_{R} \rightarrow 0^{+}$ as $R \rightarrow+\infty$.

Lemma 4.2. Let $\Omega$ satisfy $\left(1.1\right.$ with $d \geq 1$, let $e$ be in $S^{d-1}$, and assume that $\Omega$ is invariant in direction $\tilde{e}$. Let $f:[0,+\infty) \rightarrow \mathbb{R}$ be a function of class $C^{1}$ such that $f(0)=0$ and $f^{\prime}(0)>0$, and let $c$ be such that $|c|<2 \sqrt{f^{\prime}(0)}$. Then there exist $R>0$ and $\varepsilon_{0}>0$ such that, for all $\varepsilon \in\left(0, \varepsilon_{0}\right)$, there is a function $w$ satisfying

$$
\begin{cases}\Delta_{x, y} w+c \tilde{e} \cdot \nabla_{x, y} w+f(w) \geq 0 & \text { in } \Omega \cap B_{R}, \\ w>0 & \text { in } \bar{\Omega} \cap B_{R}, \\ w=0 & \text { on } \bar{\Omega} \cap \partial B_{R}, \\ \partial_{\nu} w=0 & \text { on } \partial \Omega \cap B_{R}, \\ \|w\|_{L_{\left(\Omega \cap B_{R}\right)} \leq \varepsilon .} & \end{cases}
$$

Proof. Let $R$ be fixed large enough so that the first eigenvalue $\lambda_{R}$ of (4.1) satisfies

$$
\lambda_{R}<f^{\prime}(0)-c^{2} / 4 \text {. }
$$


The latter is possible by Lemma 4.1 and since $|c|<2 \sqrt{f^{\prime}(0)}$. It then follows that the function $w(x, y)=\varepsilon e^{-c e \cdot x / 2} \psi_{R}(x, y)$ satisfies

$$
\Delta w+c \tilde{e} \cdot \nabla w+f(w)=f\left(\varepsilon e^{-c e \cdot x / 2} \psi_{R}(x, y)\right)-\left(c^{2} / 4+\lambda_{R}\right) \varepsilon e^{-c e \cdot x / 2} \psi_{R}(x, y) \geq 0
$$

in $\Omega \cap B_{R}$ for $\varepsilon>0$ small enough. On the other hand, the function $w$ is positive on $\bar{\Omega} \cap B_{R}$, vanishes on $\bar{\Omega} \cap \partial B_{R}$, has small $L^{\infty}\left(\Omega \cap B_{R}\right)$ norm for $\varepsilon$ small enough, and it satisfies the Neumann boundary condition $\partial_{\nu} w=0$ on $\partial \Omega \cap B_{R}$ because so does $\psi_{R}$ and $\Omega$ is invariant in direction $\tilde{e}$. That completes the proof of Lemma 4.2

Proof of Proposition 1.14 First of all, one can assume without loss of generality that $u \not \equiv 0$, whence the strong maximum principle yields $u>0$ in $\bar{\Omega}$. If $\Omega$ is bounded (this corresponds to the case $d=0$ ), then the minimum $m$ of $u$ in $\bar{\Omega}$ is reached and positive, and, since $g$ is positive in $(0,1)$, the strong maximum principle and Hopf lemma yield $m \geq 1$. Similarly, since $g$ is negative in $(1,+\infty)$, the maximum $M$ of $u$ satisfies $M \leq 1$. Therefore, $u \equiv 1$.

Let us now consider the general case of a domain $\Omega$ which is unbounded, i.e. $d \geq 1$. Two cases may occur:

Case 1: $m=\inf _{\bar{\Omega}} u>0$. Let $\left(x_{n}, y_{n}\right)_{n \in \mathbb{N}}$ be a sequence of points in $\bar{\Omega}$ such that $u\left(x_{n}, y_{n}\right) \rightarrow m$ as $n \rightarrow+\infty$. If $m$ is reached, then the points $\left(x_{n}, y_{n}\right)$ may be assumed to be bounded. In the general case, there exist some points $\tilde{x}_{n} \in L_{1} \mathbb{Z} \times \cdots \times L_{d} \mathbb{Z}$ such that $\left(x_{n}-\tilde{x}_{n}, y_{n}\right) \in \bar{C}$ (remember that $C$ is the periodicity cell of $\Omega$ ). Up to taking a subsequence, one can assume that $\left(x_{n}-\tilde{x}_{n}, y_{n}\right) \rightarrow(\bar{x}, \bar{y}) \in \bar{C}$ as $n \rightarrow+\infty$.

Set $u_{n}(x, y)=u\left(x+\tilde{x}_{n}, y\right)$. The functions $u_{n}$ are defined in $\Omega$, by $L$-periodicity of $\Omega$, and they satisfy the same equation (1.24) as $u$. From standard elliptic estimates and Sobolev injections, the functions $u_{n}$ converge, up to taking a subsequence, in $C_{\mathrm{loc}}^{2, \delta}(\bar{\Omega})$ (for all $0 \leq \delta<1$ ) to a function $u_{\infty}$ solving (1.24). Furthermore, $u_{\infty} \geq m$ and $u_{\infty}(\bar{x}, \bar{y})=m$. If $m<1$, then $g(m)>0$ and one gets a contradiction with the strong maximum principle and Hopf lemma. Therefore, $m \geq 1$. Similarly, one can prove that $M=\sup _{\bar{\Omega}} u \leq 1$. Hence, $u \equiv 1$.

Case 2: $m=\inf _{\bar{\Omega}} u=0$. Remember that $\left(e_{i}\right)_{1 \leq i \leq d}$ denotes the canonical basis of $\mathbb{R}^{d}$. Since $\Omega$ satisfies 1.1 , the function $(x, y) \mapsto u\left(x+L_{1} e_{1}, y\right)$ is defined in $\bar{\Omega}$. On the other hand, Harnack type inequalities imply that the function

$$
v(x, y)=\frac{u\left(x+L_{1} e_{1}, y\right)}{u(x, y)}
$$

is globally bounded in $\bar{\Omega}$. Set $M_{1}=\lim \sup _{u(x, y) \rightarrow 0,(x, y) \in \bar{\Omega}} v$ and let $\left(x_{n}, y_{n}\right) \in \bar{\Omega}$ be such that $u\left(x_{n}, y_{n}\right) \rightarrow 0$ and $v\left(x_{n}, y_{n}\right) \rightarrow M_{1}$ as $n \stackrel{+}{\rightarrow}+\infty$. Let $\tilde{x}_{n} \in L_{1} \mathbb{Z} \times \cdots \times$ $L_{d} \mathbb{Z}$ be such that $\left(x_{n}-\tilde{x}_{n}, y_{n}\right) \in \bar{C}$. Up to taking a subsequence, one can assume that $\left(x_{n}-\tilde{x}_{n}, y_{n}\right) \rightarrow(\bar{x}, \bar{y}) \in \bar{C}$ as $n \rightarrow+\infty$.

For each $n \in \mathbb{N}$, let $u_{n}$ be the function defined in $\bar{\Omega}$ by

$$
u_{n}(x, y)=\frac{u\left(x+\tilde{x}_{n}, y\right)}{u\left(x_{n}, y_{n}\right)} .
$$


From the Harnack inequalities, the functions $u_{n}$ are locally bounded in $\bar{\Omega}$. On the other hand, the functions $(x, y) \mapsto u\left(x+\tilde{x}_{n}, y\right)$ satisfy the same equation as $u$ and $u\left(x_{n}, y_{n}\right) \rightarrow$ 0 as $n \rightarrow+\infty$. From standard elliptic estimates, the functions $u_{n}$ converge in $C_{\text {loc }}^{2, \delta}(\bar{\Omega})$ (for all $0 \leq \alpha<1$ ), up to taking a subsequence, to a nonnegative function $u_{\infty}$ solving

$$
\begin{cases}\Delta u_{\infty}+b \cdot \nabla u_{\infty}+g^{\prime}(0) u_{\infty}=0 & \text { in } \Omega, \\ \partial_{\nu} u_{\infty}=0 & \text { on } \partial \Omega .\end{cases}
$$

Furthermore, $u_{\infty}(\bar{x}, \bar{y})=1$, whence $u_{\infty}>0$ in $\bar{\Omega}$ from the strong maximum principle. Owing to the definitions of $M_{1}$ and of the sequence $\left(x_{n}, y_{n}\right)$, one finds that $0<$ $u_{\infty}\left(x+L_{1} e_{1}, y\right) / u_{\infty}(x, y) \leq M_{1}$ and $u_{\infty}\left(\bar{x}+L_{1} e_{1}, \bar{y}\right) / u_{\infty}(\bar{x}, \bar{y})=M_{1}$. Notice then that $M_{1}>0$ since $u_{\infty}$ is positive in $\bar{\Omega}$.

The function

$$
\xi(x, y):=\frac{u_{\infty}\left(x+L_{1} e_{1}, y\right)}{u_{\infty}(x, y)}
$$

satisfies

$$
\begin{cases}\Delta \xi+2 \frac{\nabla u_{\infty}}{u_{\infty}} \cdot \nabla \xi+b \cdot \nabla \xi=0 & \text { in } \Omega, \\ \partial_{\nu} \xi=0 & \text { on } \partial \Omega,\end{cases}
$$

with $\xi \leq M_{1}$ in $\bar{\Omega}$ and equality at $(\bar{x}, \bar{y})$. It follows from the strong maximum principle that $\xi \equiv M_{1}$ in $\bar{\Omega}$, whence $u_{\infty}\left(x+L_{1} e_{1}, y\right) \equiv M_{1} u_{\infty}(x, y)$.

In other words, if we set $\alpha_{1}=\left(\ln M_{1}\right) / L_{1}$, the function $\varphi_{1}(x, y):=e^{-\alpha_{1} x_{1}} u_{\infty}(x, y)$ is positive in $\bar{\Omega}$ and it satisfies

$$
\begin{cases}\Delta \varphi_{1}+2 \alpha_{1} \partial_{x_{1}} \varphi_{1}+\alpha_{1}^{2} \varphi_{1}+b \cdot \nabla \varphi_{1}+b_{1} \alpha_{1} \varphi_{1}+g^{\prime}(0) \varphi_{1}=0 & \text { in } \Omega, \\ \partial_{\nu} \varphi_{1}+\alpha_{1} \nu \cdot e_{1} \varphi_{1}=0 & \text { on } \partial \Omega, \\ \varphi_{1}\left(x+L_{1} e_{1}, y\right)=\varphi_{1}(x, y) & \text { in } \Omega,\end{cases}
$$

where $b=\left(b_{1}, \ldots, b_{N}\right)$.

Notice now that the function $\varphi_{1}\left(x+L_{2} e_{2}, y\right) / \varphi_{1}(x, y)$ is globally bounded in $\bar{\Omega}$, once again from Harnack type inequalities. Then set

$$
M_{2}:=\sup _{(x, y) \in \bar{\Omega}} \frac{\varphi_{1}\left(x+L_{2} e_{2}, y\right)}{\varphi_{1}(x, y)}
$$

and follow the same procedure as before, and so on $d$ times. One then gets the existence of a positive $L$-periodic function $\varphi$ in $\bar{\Omega}$ satisfying

$$
\begin{cases}\Delta \varphi+2 \alpha \cdot \nabla_{x} \varphi+|\alpha|^{2} \varphi+b \cdot \nabla \varphi+b \cdot \tilde{\alpha} \varphi+g^{\prime}(0) \varphi=0 & \text { in } \Omega, \\ \partial_{\nu} \varphi+v \cdot \alpha \varphi=0 & \text { on } \partial \Omega\end{cases}
$$

for some $\alpha=\left(\alpha_{1}, \ldots, \alpha_{d}\right) \in \mathbb{R}^{d}$, where $|\alpha|^{2}=\alpha_{1}^{2}+\cdots+\alpha_{d}^{2}, \tilde{\alpha}=\left(\alpha_{1}, \ldots, \alpha_{d}, 0, \ldots, 0\right)$ $\in \mathbb{R}^{N}$ and $\nabla$ is the gradient with respect to both $(x, y)$ variables. 
Divide the above equation by $\varphi$ and integrate by parts over the cell $C$. By periodicity, it follows that

$$
\int_{C}\left\{\frac{|\nabla \varphi|^{2}}{\varphi^{2}}+2 \alpha \cdot \frac{\nabla_{x} \varphi}{\varphi}+|\alpha|^{2}+b \cdot \frac{\nabla \varphi}{\varphi}+b \cdot \tilde{\alpha}+g^{\prime}(0)\right\}=0 .
$$

In other words,

$$
\int_{C}\left\{\left|\frac{\nabla \varphi}{\varphi}+\tilde{\alpha}+\frac{b}{2}\right|^{2}+\left(g^{\prime}(0)-\frac{|b|^{2}}{4}\right)\right\}=0 .
$$

One then gets a contradiction with the assumption $|b|<2 \sqrt{g^{\prime}(0)}$.

As a conclusion, Case 2 is ruled out, whence $\inf _{\bar{\Omega}} u>0$ and $u \equiv 1$.

Remark 4.3. In the case where $\Omega=\mathbb{R}^{N}$, the above proof can be slightly simplified. Indeed, by Lemma 4.2, there is $R>0$ and a function $w$ such that $w>0$ in $B_{R}, w=0$ on $\partial B_{R}, w<u$ in $\bar{B}_{R}$ and $\Delta w+c \tilde{e} \cdot \nabla w+f(w) \geq 0$ in $B_{R}$. Since the equation 1.24 satisfied by $u$ is translation invariant, and since $u>0$ in $\mathbb{R}^{N}$, one can slide $u$ in any direction and prove that, for all $x_{0} \in \mathbb{R}^{N}, w<u\left(\cdot+x_{0}\right)$ in $\bar{B}_{R}$. Therefore, $\inf _{\mathbb{R}^{N}} u>0$ and one concludes as in Case 1 of the above proof that $u \equiv 1$.

This result in the case $\Omega=\mathbb{R}^{N}$ has been known since the paper of Aronson and Weinberger [2], who used parabolic tools. The above arguments actually provide a simpler proof using elliptic arguments.

Proof of Theorem 1.13 As already emphasized, it only remains to prove that, if $\Omega$ is a straight cylinder in direction $\tilde{e}$, then $w^{*}(e)=2 \sqrt{f^{\prime}(0)}$ for any nonnegative continuous and compactly supported initial condition $u_{0} \neq \equiv 0$.

Let $u_{0}$ be such a function. Since $w^{*}(e) \leq c^{*}(e) \leq 2 \sqrt{f^{\prime}(0)}$, one only has to prove that $w^{*}(e) \geq 2 \sqrt{f^{\prime}(0)}$.

Let $0 \leq c<2 \sqrt{f^{\prime}(0)}$ and let us actually prove that $u(t, x+c t e, y) \rightarrow 1$ as $t \rightarrow+\infty$ locally in $(x, y) \in \bar{\Omega}$. Let us mention that, since $\Omega$ is invariant in direction $\tilde{e}$, the function $(x, y) \mapsto v(t, x, y)=u(t, x+c t e, y)$ is defined in $\bar{\Omega}$ for all $t \geq 0$. The function $v$ actually satisfies

$$
v_{t}=\Delta v+c \tilde{e} \cdot \nabla v+f(v)
$$

for all $t>0$ and $(x, y) \in \bar{\Omega}$, together with Neumann boundary conditions on $\partial \Omega$, and initial condition $u_{0}$. Notice that $v(t, x, y)>0$ for all $t>0$ and $(x, y) \in \bar{\Omega}$ from the strong maximum principle.

Set $M=\sup _{\mathbb{R}^{N}} u_{0}$. One has $0<M<+\infty$ by assumption. Let $\xi(t)$ be the function solving $\dot{\xi}=f(\xi)$ and $\xi(0)=M$. From the assumptions on $f$, one has $\xi(t) \rightarrow 1$ as $t \rightarrow+\infty$. Furthermore, $v(t, x, y) \leq \xi(t)$ for all $t \geq 0$ and $(x, y) \in \bar{\Omega}$ by the parabolic maximum principle. Therefore,

$$
\limsup _{t \rightarrow+\infty} \sup _{(x, y) \in \bar{\Omega}} v(t, x, y) \leq 1 .
$$

On the other hand, from Lemma 4.2 there exists $R>0$ (large enough so that $\left.B_{R} \cap \Omega \neq \emptyset\right)$ and a function $w$ solving (4.2) and such that, say, $v(1, x, y) \geq w(x, y)$ 
for all $(x, y) \in \bar{B}_{R} \cap \bar{\Omega}$ (remember that $\min _{(x, y) \in \bar{B}_{R} \cap \bar{\Omega}} v(1, x, y)>0$ ). Let $\tilde{w}$ be the function defined in $\bar{\Omega}$ by $\tilde{w}=w$ in $B_{R} \cap \bar{\Omega}$ and $\tilde{w}=0$ in $\bar{\Omega} \backslash B_{R}$. The function $\tilde{w}$ is then a subsolution for the equation satisfied by $v$. Therefore, the function $\theta$ solving

$$
\left\{\begin{array}{l}
\theta_{t}=\Delta \theta+c \tilde{e} \cdot \nabla \theta+f(\theta), \quad t>0,(x, y) \in \Omega, \\
\partial_{\nu} \theta=0, \quad t>0,(x, y) \in \partial \Omega \\
\theta(0, x, y)=\tilde{w}(x, y)
\end{array}\right.
$$

is nondecreasing with respect to $t$ and, since $\tilde{w} \leq v(1, \cdot, \cdot)$ in $\bar{\Omega}$, the function $\theta$ satisfies

$$
\forall t \geq 0, \forall(x, y) \in \bar{\Omega}, \quad \theta(t, x, y) \leq v(t+1, x, y) .
$$

Without loss of generality, one can assume that $\tilde{w} \leq 1$ in $\bar{\Omega}$, whence $\theta(t, x, y) \leq 1$ for all $(t, x, y) \in \mathbb{R}^{+} \times \bar{\Omega}$. By monotonicity in $t$, the function $\theta(t, x, y)$ converges as $t \rightarrow+\infty$ to a function $\psi(x, y)$. From standard parabolic estimates, the convergence $\theta(t, x, y) \rightarrow \psi(x, y)$ as $t \rightarrow+\infty$ holds locally uniformly in $\bar{\Omega}$ and the function $\psi$ is a classical solution of 1.24 with $g=f, b=c \tilde{e}$ and $|b|<2 \sqrt{f^{\prime}(0)}$. Furthermore, $0 \leq \tilde{w} \leq \psi \leq 1$ in $\bar{\Omega}$ (thus, $\psi \not \equiv 0$ since $w>0$ in $B_{R} \cap \Omega \neq \emptyset$ ). Proposition 1.14 yields $\psi \equiv 1$.

One deduces from 4.4 that $\liminf _{t \rightarrow+\infty} \min _{(x, y) \in K} v(t, x, y) \geq 1$ for all compact sets $K \subset \bar{\Omega}$.

One concludes from 4.3 that $v(t, x, y) \rightarrow 1$ as $t \rightarrow+\infty$ locally in $(x, y) \in \bar{\Omega}$. That completes the proof of Theorem 1.13

Remark 4.4. If $\Omega$ is invariant in direction $\tilde{e}$ and satisfies $(1.1)$, then $u(t, x+c t e, y) \rightarrow 1$ as $t \rightarrow+\infty$ locally in $(x, y) \in \bar{\Omega}$ for $0 \leq c<2 \sqrt{f^{\prime}(0)}$ and for all solutions $u$ of $(1.15$ ) with continuous bounded nonnegative initial condition $u_{0} \not \equiv 0$. The latter indeed holds from the maximum principle even if $u_{0}$ is not compactly supported. Furthermore, under the additional assumption that $u_{0}$ is compactly supported, then $u(t, x+c t e, y) \rightarrow 0$ as $t \rightarrow+\infty$ locally in $(x, y) \in \bar{\Omega}$ for all $c>2 \sqrt{f^{\prime}(0)}$.

\section{References}

[1] Aronson, D. G., Weinberger, H. F.: Nonlinear diffusion in population genetics, combustion and nerve propagation. In: Partial Differential Equations and Related Topics, Lecture Notes in Math. 446, Springer, New York, 5-49 (1975) Zbl 0325.35050 MR 0427837

[2] Aronson, D. G., Weinberger, H. F.: Multidimensional nonlinear diffusions arising in population genetics. Adv. Math. 30, 33-76 (1978) Zbl 0407.92014 MR 0511740

[3] Audoly, B., Berestycki, H., Pomeau, Y.: Réaction-diffusion en écoulement stationnaire rapide. C. R. Acad. Sci. Paris Sér. II 328, 255-262 (2000) Zbl 0992.76097

[4] Bates, P. W., Fife, P. C., Ren, X., Wang, X.: Traveling waves in a convolution model for phase transitions. Arch. Rat. Mech. Anal. 138, 105-136 (1997) Zbl 0889.45012 MR 1463804

[5] Benguria, R. D., Depassier, M. C.: Variational characterization of the speed of propagation of fronts for the nonlinear diffusion equation. Comm. Math. Phys. 175, 221-227 (1996) Zbl 0856.35058 MR 1370094 
[6] Berestycki, H.: The influence of advection on the propagation of fronts in reaction-diffusion equations. In: Nonlinear PDE's in Condensed Matter and Reactive Flows, H. Berestycki and Y. Pomeau (eds.), Kluwer, 1-45 (2002)

[7] Berestycki, H., Hamel, F.: Front propagation in periodic excitable media. Comm. Pure Appl. Math. 55, 949-1032 (2002) Zbl 1024.37054 MR 1900178

[8] Berestycki, H., Hamel, F.: Gradient estimates for elliptic regularizations of semilinear parabolic and degenerate elliptic equations. Comm. Partial Differential Equations 30, 139$156(2005)$

[9] Berestycki, H., Hamel, F., Nadirashvili, N.: The principal eigenvalue of elliptic operators with large drift and applications to nonlinear propagation phenomena. Comm. Math. Phys. 253, 451-480 (2005)

[10] Berestycki, H., Hamel, F., Nadirashvili, N.: The speed of propagation for KPP type problems. I: General domains. Preprint

[11] Berestycki, H., Hamel, F., Roques, L.: Analysis of the periodically fragmented environment model. I: Influence of periodic heterogeneous environment on species persistence. J. Math. Biology, to appear

[12] Berestycki, H., Hamel, F., Roques, L.: Analysis of the periodically fragmented environment model. II: Biological invasions and pulsating travelling fronts. J. Math. Pures Appl., to appear (2005)

[13] Berestycki, H., Larrouturou, B., Lions, P.-L.: Multidimensional traveling-wave solutions of a flame propagation model. Arch. Rat. Mech. Anal. 111, 33-49 (1990) Zbl 0711.35066 MR 1051478

[14] Berestycki, H., Larrouturou, B., Roquejoffre, J.-M.: Stability of traveling fronts in a curved flame model, Part I, Linear analysis. Arch. Rat. Mech. Anal. 117, 97-117 (1992) Zbl 0763.76033 MR 1145107

[15] Berestycki, H., Nicolaenko, B., Scheurer, B.: Traveling wave solutions to combustion models and their singular limits. SIAM J. Math. Anal. 16, 1207-1242 (1985) Zbl 0596.76096 MR 0807905

[16] Berestycki, H., Nirenberg, L.: On the method of moving planes and the sliding method. Bol. Soc. Bras. Mat. 22, 1-37 (1991) Zbl 0784.35025 MR 1159383

[17] Berestycki, H., Nirenberg, L.: Travelling fronts in cylinders. Ann. Inst. H. Poincaré Anal. Non Linéaire 9, 497-572 (1992) Zbl 0799.35073 MR 1191008

[18] Berestycki, H., Nirenberg, L., Varadhan, S. R. S.: The principal eigenvalue and maximum principle for second order elliptic operators in general domains. Comm. Pure Appl. Math. 47, 47-92 (1994) Zbl 0806.35129 MR 1258192

[19] Bonnet, A., Hamel, F.: Existence of non-planar solutions of a simple model of premixed Bunsen flames. SIAM J. Math. Anal. 31, 80-118 (1999) Zbl 0942.35072 MR 1742304

[20] Bramson, M.: Convergence of solutions of the Kolmogorov equation to travelling waves. Mem. Amer. Math. Soc. 44 (1983) Zbl 0517.60083 MR 0705746

[21] Caffarelli, L. A., Lee, K.-A., Mellet, A.: Singular limit and homogenization for flame propagation in periodic excitable media. Preprint

[22] Capdeboscq, Y.: Homogenization of a neutronic critical diffusion problem with drift. Proc. Roy. Soc. Edinburgh Sect. A 132, 567-594 (2002) Zbl pre01790831 MR 1912416

[23] Conca, C., Vanninathan, M.: Homogenization of periodic structures via Bloch decomposition. SIAM J. Appl. Math. 57, 1639-1659 (1997) Zbl 0990.35019 MR 1484944

[24] Constantin, P., Kiselev, A., Oberman, A., Ryzhik, L.: Bulk burning rate in passive-reactive diffusion. Arch. Rat. Mech. Anal. 154, 53-91 (2000) Zbl 0979.76093 MR 1778121

[25] Constantin, P., Kiselev, A., Ryzhik, L.: Quenching of flames by fluid advection. Comm. Pure Appl. Math. 54, 1320-1342 (2001) Zbl 1032.35087 MR 1846800 
[26] Coutinho, R., Fernandez, B.: Fronts and interfaces in bistable extended mappings. Nonlinearity 11, 1407-1433 (1998) Zbl 0919.58022 MR 1644421

[27] Diekmann, O.: Run for your life. A note on the asymptotic speed of propagation of an epidemic. J. Differential Equations 33, 58-73 (1979) Zbl 0377.45007 MR 0540817

[28] Ebert, U., van Saarloos, W.: Front propagation into unstable states: universal algebraic convergence towards uniformly translating pulled fronts. Phys. D 146, 1-99 (2000) Zbl 0984.35030 MR 1787406

[29] Fannjiang, A., Papanicolaou, G.: Convection enhanced diffusion for periodic flows. SIAM J. Appl. Math. 54, 333-408 (1994) Zbl 0796.76084 MR 1265233

[30] Fife, P. C.: Mathematical Aspects of Reacting and Diffusing Systems. Lecture Notes in Biomath. 28, Springer (1979) Zbl 0403.92004 MR 0527914

[31] Fife, P. C., McLeod, J. B.: The approach of solutions of non-linear diffusion equations to traveling front solutions. Arch. Rat. Mech. Anal. 65, 335-361 (1977) Zbl 0361.35035 MR 0442480

[32] Fisher, R. A.: The wave of advance of advantageous genes. Ann. Eugenics 7, 335-369 (1937) JFM 63.1111.04

[33] Freidlin, M.: Propagation of a concentration wave in the presence of random motion associated with the growth of a substance. Soviet Math. Dokl. 20, 503-507 (1979) MR 0534050

[34] Freidlin, M.: On wave front propagation in periodic media. In: Stochastic Analysis and Applications, M. Pinsky (ed.), Adv. Probab. Related Topics 7, Dekker, New York, 147-166 (1984) Zbl 0549.60054 MR 0776979

[35] Freidlin, M.: Functional Integration and Partial Differential Equations. Ann. of Math. Stud. 109, Princeton Univ. Press (1985) Zbl 0568.60057 MR 0833742

[36] Freidlin, M.: Wave front propagation for KPP-type equations. In: Surveys in Appl. Math. 2, Plenum, New York, 1-62 (1995) Zbl 0848.60065 MR 1387615

[37] Gallay, T.: Local stability of critical fronts in nonlinear parabolic pde's. Nonlinearity 7, 741764 (1994) Zbl 0801.35046 MR 1275528

[38] Gardner, R. A.: Existence of multidimensional travelling wave solutions of an initial-boundary value problem. J. Differential Equations 61, 335-379(1986) Zbl 0549.35066 MR 0829368

[39] Gärtner, J., Freidlin, M.: On the propagation of concentration waves in periodic and random media. Soviet Math. Dokl. 20, 1282-1286 (1979) Zbl 0447.60060 MR 0553200

[40] Hadeler, K.P., Rothe, F.: Travelling fronts in nonlinear diffusion equations. J. Math. Biol. 2, 251-263 (1975) Zbl 0343.92009 MR 0411693

[41] Hamel, F.: Formules min-max pour les vitesses d'ondes progressives multidimensionnelles. Ann. Fac. Sci. Toulouse 8, 259-280 (1999) Zbl 0956.35041 MR 1751443

[42] Hamel, F., Monneau, R.: Solutions of semilinear elliptic equations in $\mathbb{R}^{N}$ with conicalshaped level sets. Comm. Partial Differential Equations 25, 769-819 (2000) Zbl 0952.35041 MR 1759793

[43] Hamel, F., Nadirashvili, N.: Travelling waves and entire solutions of the Fisher-KPP equation in $\mathbb{R}^{N}$. Arch. Rat. Mech. Anal. 157, 91-163 (2001) Zbl 0987.35072 MR 1830037

[44] Hamel, F., Omrani, S.: Existence of multidimensional travelling fronts with a multistable nonlinearity. Adv. Differential Equations 5, 557-582 (2000) Zbl pre01700742 MR 1750111

[45] Harris, G., Hudson, W., Zinner, B.: Traveling wavefronts for the discrete Fisher's equation. J. Differential Equation 105, 46-62 (1993) Zbl 0778.34006 MR 1237977

[46] Heinze, S.: Homogenization of flame fronts. Preprint IWR, Heidelberg (1993)

[47] Heinze, S.: Wave solution for reaction-diffusion systems in perforated domains. Z. Anal. Anwendungen 20, 661-670 (2001) Zbl 0986.35051 MR 1863939

[48] Heinze, S.: The speed of travelling waves for convective reaction-diffusion equations. Preprint MPI, Leipzig (2001) 
[49] Heinze, S., Papanicolaou, G., Stevens, A.: Variational principles for propagation speeds in inhomogeneous media. SIAM J. Appl. Math. 62, 129-148 (2001) Zbl 0995.35031 MR 1857539

[50] Hudson, W., Zinner, B.: Existence of travelling waves for reaction-diffusion equations of Fisher type in periodic media. In : Boundary Problems for Functional Differential Equations, World Sci., 187-199 (1995) Zbl 0846.35062 MR 1375475

[51] Jones, C. K. R. T.: Spherically symmetric solutions of a reaction-diffusion equation. J. Differential Equations 49, 142-169 (1983) Zbl 0523.35059 MR 0704268

[52] Kanel', Ya. I.: Certain problems of burning-theory equations. Soviet Math. Dokl. 2, 48-51 (1961) Zbl 0138.35103 MR 0117429

[53] Kanel', Ya. I.: On the stability of solutions of the equations of combustion theory for finite initial functions. Mat. Sb. 65, 398-413 (1964) (in Russian) MR 0177209

[54] Kan-On, Y.: Parameter dependence of propagation speed of travelling waves for competitiondiffusion equations. SIAM J. Math. Anal. 26, 340-363 (1995)

[55] Kinezaki, N., Kawasaki, K., Takasu, F., Shigesada, N.: Modeling biological invasion into periodically fragmented environments. Theor. Population Biology 64, 291-302 (2003)

[56] Kiselev, A., Ryzhik, L.: Enhancement of the traveling front speeds in reaction-diffusion equations with advection. Ann. Inst. H. Poincaré Anal. Non Linéaire 18, 309-358 (2001) Zbl 1002.35069 MR 1831659

[57] Kolmogorov, A. N., Petrovsky, I. G., Piskunov, N. S.: Étude de l'équation de la diffusion avec croissance de la quantité de matière et son application à un problème biologique. Bull. Univ. Moscou Sér. Internat. A 1, 1-26 (1937); English transl. in: Dynamics of Curved Fronts, P. Pelcé (ed.), Academic Press, 105-130 (1988) Zbl 0018.32106

[58] Kozlov, S., Piatnitski, A. L.: Degeneration of effective diffusion in the presence of periodic potential. Ann. Inst. H. Poincaré Anal. Non Linéaire 32, 571-587 (1996) Zbl 0888.35013 MR 1411272

[59] Lau, K.-S.: On the nonlinear diffusion equation of Kolmogorov, Petrovsky, and Piscounov. J. Differential Equations 59, 44-70 (1985) Zbl 0584.35091 MR 0803086

[60] Levermore, C. D., Xin, J. X.: Multidimensional stability of traveling waves in a bistable reaction-diffusion equation, II. Comm. Partial Differential Equations 17, 1901-1924 (1999) Zbl 0789.35020 MR 1194744

[61] Lou, B., Matano, H.: Preprint

[62] Lui, R.: Biological growth and spread modeled by systems of recursions. I. Mathematical theory. Math. Bios. 93, 269-295 (1989) Zbl 0706.92014 MR 0984281

[63] Majda, A. J., Souganidis, P. E.: Large scale front dynamics for turbulent reaction-diffusion equations with separated velocity scales. Nonlinearity 7, 1-30 (1994) Zbl 0839.76093 MR 1260130

[64] Majda, A. J., Souganidis, P. E.: Flame fronts in a turbulent combustion model with fractal velocity fields. Comm. Pure Appl. Math. 51, 1337-1348 (1998) Zbl 0939.35097 MR 1639220

[65] Mallordy, J.-F., Roquejoffre, J.-M.: A parabolic equation of the KPP type in higher dimensions. SIAM J. Math. Anal. 26, 1-20 (1995) Zbl 0813.35041 MR 1311879

[66] Matano, H.: Travelling waves in spatially inhomogeneous diffusive media - the non-periodic case. Preprint

[67] McKean, H. P.: Application of Brownian motion to the equation of KPP. Comm. Pure Appl. Math. 28, 323-331 (1975) Zbl 0316.35053 MR 0400428

[68] Mollison, D.: The rate of spatial propagation of simple epidemics. In: Proceedings of the Sixth Berkeley Symposium on Mathematical Statistics and Probability, Vol. 3, Univ. of California Press, 579-614 (1972) Zbl 0266.92008 MR 0401222

[69] Murray, J. D.: Mathematical Biology. Springer (1989) Zbl 0682.92001 MR 1007836 
[70] Namah, G., Roquejoffre, J.-M.: Convergence to periodic fronts in a class of semilinear parabolic equations. Nonlinear Differential Equations Appl. 4, 521-536 (1997) Zbl 0887.35070 MR 1485736

[71] Nakamura, K.-I.: Effective speed of traveling wavefronts in periodic inhomogeneous media. Preprint

[72] Papanicolaou, G. C., Varadhan, S. R. S.: Diffusion in regions with many small holes. In: Stochastic Differential Systems (Vilnius, 1978), Lecture Notes in Control Inform. Sci. 25, Springer, Berlin, 190-206 (1980) Zbl 0485.60076 MR 0609184

[73] Papanicolaou, G., Xin, X.: Reaction-diffusion fronts in periodically layered media. J. Statist. Phys. 63, 915-931 (1991) MR 1116041

[74] Protter, M., Weinberger, H. F.: Maximum Principles in Differential Equations. Prentice-Hall, Englewood Cliffs (1967) Zbl 0153.13602 MR 0219861

[75] Ronney, P.: Some open issues in premixed turbulent combustion. In: Mathematical Modelling in Combustion Science, J. Buckmaster and T. Takeno (eds.), Lecture Notes in Phys. 449, Springer, Berlin, 3-22 (1995)

[76] Roquejoffre, J.-M.: Stability of travelling fronts in a curved flame model, Part II: Nonlinear orbital stability. Arch. Rat. Mech. Anal. 117, 119-153 (1992) Zbl 0763.76034 MR 1145107

[77] Roquejoffre, J.-M.: Eventual monotonicity and convergence to travelling fronts for the solutions of parabolic equations in cylinders. Ann. Inst. H. Poincaré Anal. Non Linéaire 14, 499-552 (1997) Zbl 0884.35013 MR 1464532

[78] Rothe, F.: Convergence to pushed fronts. Rocky Mountain J. Math. 11, 617-633 (1981) Zbl 0516.35013 MR 0639447

[79] Sattinger, D. H.: Stability of waves of nonlinear parabolic systems. Adv. Math. 22, 312-355 (1976) Zbl 0344.35051 MR 0435602

[80] Schwetlick, H. R.: Travelling fronts for multidimensional nonlinear transport equations. Ann. Inst. H. Poincaré Anal. Non Linéaire 17, 523-550 (2000) Zbl 0965.45012 MR 1782743

[81] Shigesada, N., Kawasaki, K.: Biological Invasions: Theory and Practice. Oxford Series in Ecology and Evolution, Oxford Univ. Press., Oxford (1997)

[82] Shigesada, N., Kawasaki, K., Teramoto, E.: Spatial segregation of interacting species. J. Theoret. Biol. 79, 83-99 (1979)

[83] Stokes, A. N.: Nonlinear diffusion waveshapes generated by possible finite multiple disturbances. J. Math. Anal. Appl. 61, 370-381 (1977) Zbl 0391.35040 MR 0466976

[84] Thieme, H. R.: Asymptotic estimates of the solutions of nonlinear integral equations and asymptotic speeds for the spread of populations. I. J. Reine Angew. Math. 306, 94-121 (1979) Zbl 0395.45010 MR 0524650

[85] Uchiyama, K.: The behavior of solutions of some semilinear diffusion equation for large time. J. Math. Kyoto Univ. 18, 453-508 (1978) Zbl 0408.35053 MR 0509494

[86] Vega, J. M.: Multidimensional travelling fronts in a model from combustion theory and related problems. Differential Integral Equations 6, 131-153 (1993) Zbl 0786.35080

[87] Volpert, A. I., Volpert, V. A., Volpert, V. A.: Traveling Wave Solutions of Parabolic Systems. Transl. Math. Monogr. 140, Amer. Math. Soc. (1994) Zbl 1001.35060 MR 1297766

[88] Weinberger, H. F.: Long time behavior of a class of biological models. SIAM J. Math. Anal. 13, 353-396 (1982) Zbl 0529.92010 MR 0653463

[89] Weinberger, H. F.: On spreading speeds and traveling waves for growth and migration in periodic habitat. J. Math. Biol. 45, 511-548 (2002) Zbl pre01903690 MR 1943224

[90] Williams, F.: Combustion Theory. Addison-Wesley, Reading, MA (1983) 
[91] Xin, X.: Existence and uniqueness of travelling waves in a reaction-diffusion equation with combustion nonlinearity. Indiana Univ. Math. J. 40, 985-1008 (1991) Zbl 0727.35070 MR 1129338

[92] Xin, X.: Existence and stability of travelling waves in periodic media governed by a bistable nonlinearity. J. Dynam. Differential Equations 3, 541-573 (1991) Zbl 0769.35033 MR 1129560

[93] Xin, X.: Existence of planar flame fronts in convective-diffusive periodic media. Arch. Rat. Mech. Anal. 121, 205-233 (1992) Zbl 0764.76074 MR 1188981

[94] Xin, J.: Front propagation in heterogeneous media. SIAM Rev. 42, 161-230 (2000) Zbl 0951.35060 MR 1778352

[95] Zeldovich, Y. B., Barenblatt, G. I., Libovich, V. B., Mackviladze, G. M.: The Mathematical Theory of Combustion and Explosions. Cons. Bureau, New York (1985) MR 0781350 